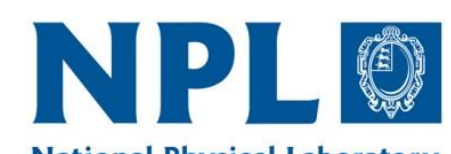

National Physical Laboratory

NPL REPORT TQE 14

COMPARISON OF VECTOR NETWORK ANALYSER (VNA)

CALIBRATION TECHNIQUES AT MICROWAVE FREQUENCIES

D SINGH, M J SALTER AND N M RIDLER

DECEMBER 2019 



\title{
COMPARISON OF VECTOR NETWORK ANALYSER (VNA) CALIBRATION TECHNIQUES AT MICROWAVE FREQUENCIES
}

\author{
D Singh, M J Salter and N M Ridler \\ Electromagnetic \& Electrochemical Technologies Department
}

\section{EXECUTIVE SUMMARY}

This report describes a comparison of some of the most commonly used calibration techniques for the accurate measurement of scattering parameters using a Vector Network Analyser system in coaxial line at microwave frequencies. The report describes the different calibration methods and their applications and compares measured S-parameters for a number of different devices measured using the same VNA calibrated with the different methods. The investigation was carried out in precision $3.5 \mathrm{~mm}$ coaxial line over the frequency range $100 \mathrm{MHz}$ to $26 \mathrm{GHz}$.

The following calibration techniques were investigated: TRL, SOLT, SOLR and ECal. A set of one-port and two-port devices with a range of S-parameter values were measured. To obtain the most comprehensive set of measurements, one-port and two-port devices with linear reflection coefficient magnitudes ranging from 0 to 1 and two-port devices with linear transmission coefficient magnitudes ranging from 0 to 1 were used.

For each calibration type, the complete set of DUTs were measured six times with a re-calibration each time. From the six repeat measurements, the mean and experimental standard deviation for the magnitude and phase of each S-parameter were computed at each frequency and were used to estimate, respectively, the value of the quantity and the variability due to random errors in the measurement of the quantity. The analysis was performed on reflection coefficients for the one-port devices and on both reflection and transmission coefficients for the two-port devices.

The measured data for all the DUTs obtained using all the calibration methods is presented in this report.

The results from this investigation show the typical experimental standard deviations to be expected in microwave S-parameter measurements for different calibration techniques and different types of DUT. This information on the performance of the various calibration methods should, to some extent, be applicable to other users and other measurement systems. 
NPL Report TQE 14

(C) NPL Management Limited, 2019

ISSN 1754-2995

https://doi.org/10.47120/npl.TQE14

National Physical Laboratory

Hampton Road, Teddington, Middlesex, TW11 OLW

Extracts from this report may be reproduced provided the source is acknowledged and the extract is not taken out of context.

Approved on behalf of NPLML by

Andrew Smith, Interim Department Head 


\section{CONTENTS}

\section{EXECUTIVE SUMMARY}

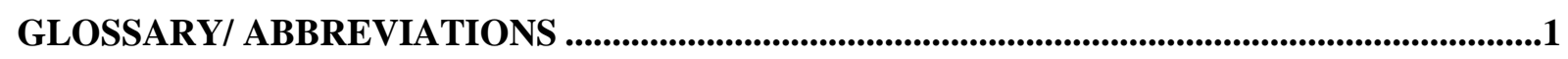

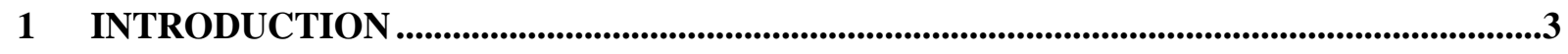

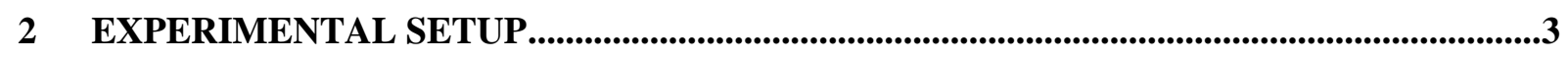

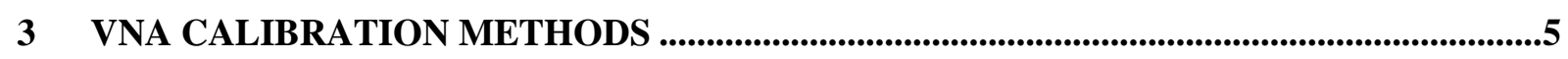

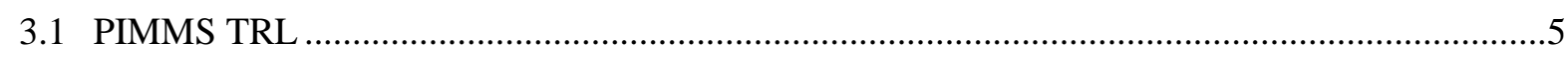

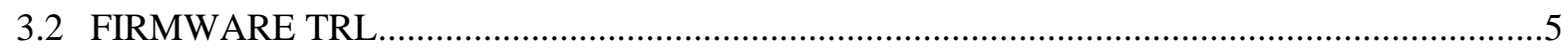

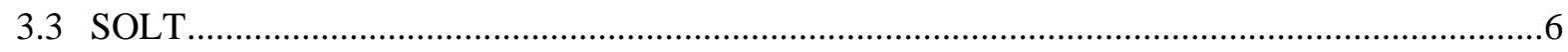

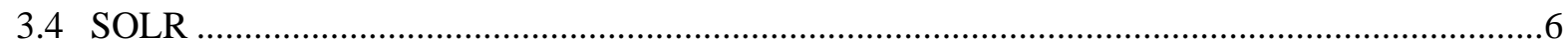

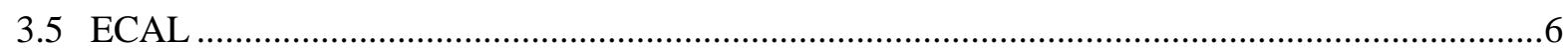

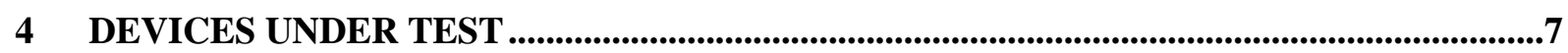

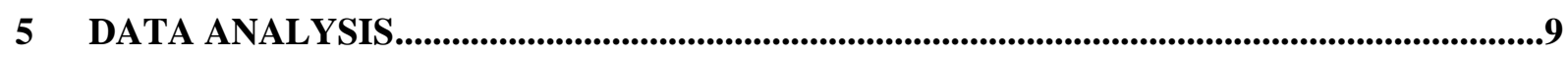

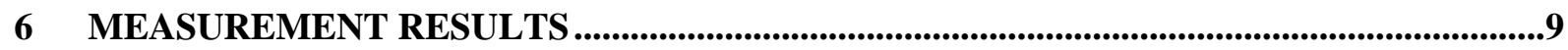

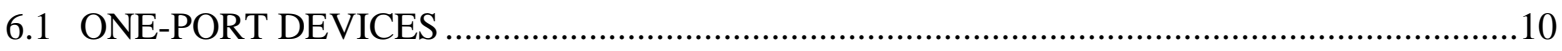

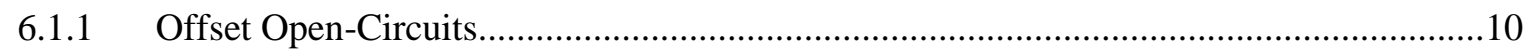

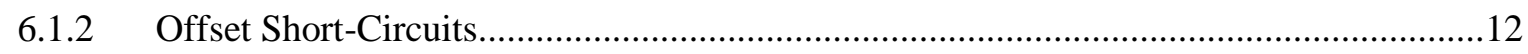

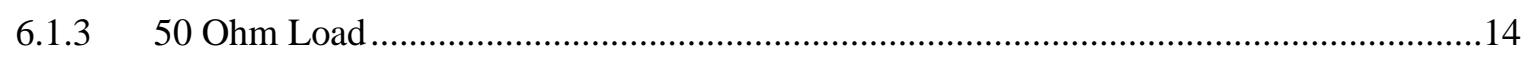

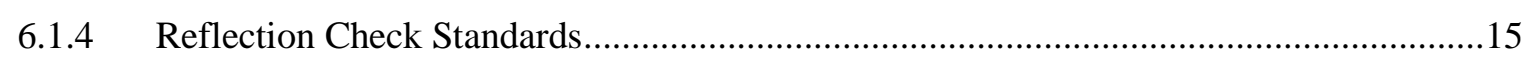

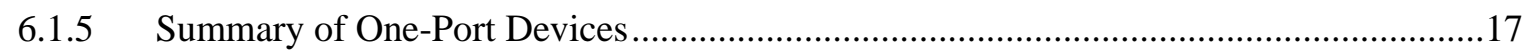

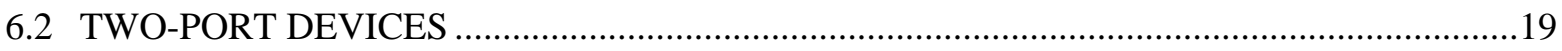

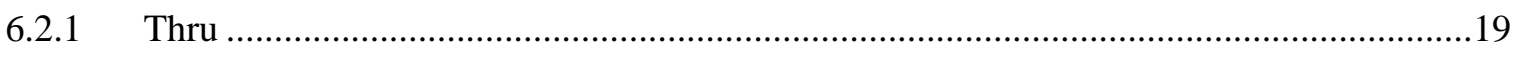

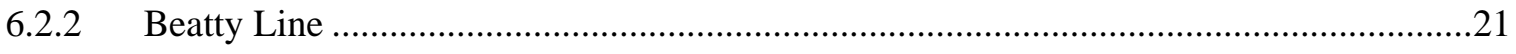

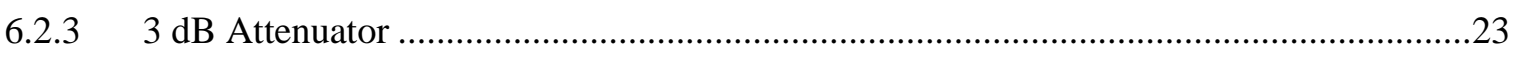

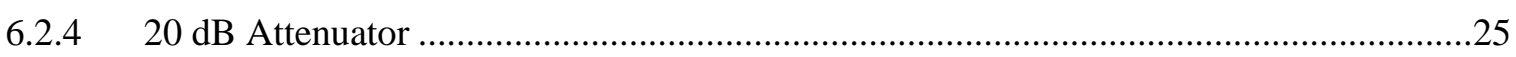

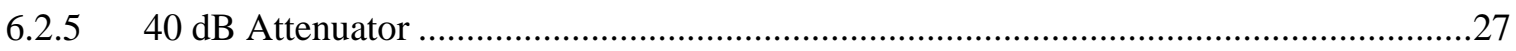

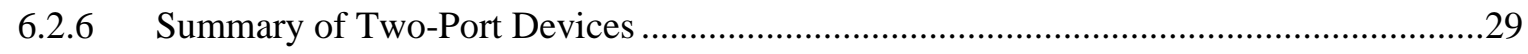

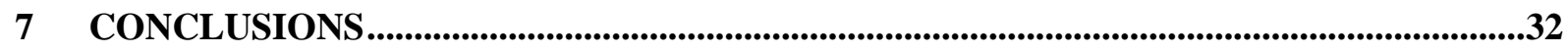

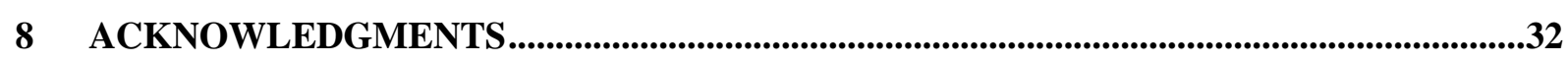

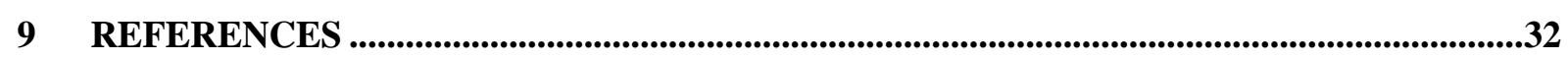


NPL Report TQE 14 


\section{GLOSSARY/ ABBREVIATIONS}

dB decibel (used for logarithmic magnitude of S-parameters)

DUT Device Under Test

ECal An electronic calibration module used to calibrate a VNA

F Female connector

M Male connector

mU milliunit (used for linear magnitude of S-parameters)

PIMMS NPL's Primary Impedance Microwave Measurement System

SD Standard Deviation

SOLR Short Open Load Reciprocal method of VNA calibration (also known as the unknown thru method)

SOLT Short Open Load Thru method of VNA calibration

TRL Thru Reflect Line method of VNA calibration

VNA Vector Network Analyser 
NPL Report TQE 14 


\section{INTRODUCTION}

The performance of a high frequency device is usually measured using a Vector Network Analyser (VNA) which measures the complex-valued scattering parameters (S-parameters) of the device i.e. the magnitudes and phases of its reflection and transmission coefficients.

Before a device under test (DUT) can be measured using a VNA, the VNA and the associated cables and adaptors etc. need to be calibrated to remove systematic errors. In this report, six different VNA calibration methods are compared by measuring various passive DUTs having a wide range of reflection and transmission coefficient values.

The VNA calibration methods compared in this investigation were:

- TRL calibration implemented using NPL's PIMMS system [1]

- TRL calibration implemented using the VNA firmware

- SOLT calibration

- SOLR calibration with a zero length (flush) thru

- SOLR calibration with a Reciprocal (non-zero length) thru

- ECal calibration

The DUTs were:

- Two offset open-circuits (male and female with different offset lengths)

- Two offset short-circuits (male and female with different offset lengths)

- A $50 \Omega$ load (male)

- Two reflection check standards (male and female 1-port devices with well-characterized reflection coefficients)

- A thru connection (direct connection of the VNA test ports)

- A Beatty line

- A $3 \mathrm{~dB}$ fixed attenuator

- A $20 \mathrm{~dB}$ fixed attenuator

- A $40 \mathrm{~dB}$ fixed attenuator

\section{EXPERIMENTAL SETUP}

For each of the calibration methods, six sets of measurements were made where each set of measurements consisted of a calibration of the VNA followed by measurement of the S-parameters of each of the DUTs.

The DUTs used for the investigation were one-port and two-port devices fitted with precision $3.5 \mathrm{~mm}$ coaxial connectors. According to [2], $3.5 \mathrm{~mm}$ connectors operate mode-free up to at least $33 \mathrm{GHz}$. However, $3.5 \mathrm{~mm}$ calibration kits from most venders such as Keysight, Maury Microwaves etc. are usually only characterised and supported to $26.5 \mathrm{GHz}$. Therefore, the investigation in this report was performed between $0.1 \mathrm{GHz}$ and $26 \mathrm{GHz}$.

Further details of the VNA calibration methods and DUTs are given in Sections 3 and 4 respectively.

The measurements were made using a four-port Keysight N5247A PNA-X VNA which has a frequency range of $10 \mathrm{MHz}$ to $67 \mathrm{GHz}$ and is fitted with $1.85 \mathrm{~mm}$ coaxial connectors. To allow the DUTs to be 
connected to the VNA, $1.85 \mathrm{~mm}$ to $3.5 \mathrm{~mm}$ adaptors were used on VNA test ports 1 and 2. A flexible coaxial cable with good amplitude and phase stability was used on VNA test port 2. Port 1 of the VNA consisted of a $3.5 \mathrm{~mm}$ male test port and port 2 of the VNA consisted of a $3.5 \mathrm{~mm}$ female test port.

The VNA settings used during the measurements were as follows:

1. Frequency range: $100 \mathrm{MHz}$ to $26 \mathrm{GHz}$

2. Frequency step: $50 \mathrm{MHz}$ below $1 \mathrm{GHz}$ and $0.2 \mathrm{GHz}$ above $1 \mathrm{GHz}$

3. Power: $-5 \mathrm{dBm}$

4. IF Bandwidth: $10 \mathrm{~Hz}$

5. Averaging Factor: 2

The measurements were carried out in a temperature-controlled laboratory at a temperature of $23{ }^{\circ} \mathrm{C} \pm 2{ }^{\circ} \mathrm{C}$. A photograph of the measurement set-up is shown in Figure 1. A photograph of a twoport DUT connected to the VNA is shown in Figure 2.

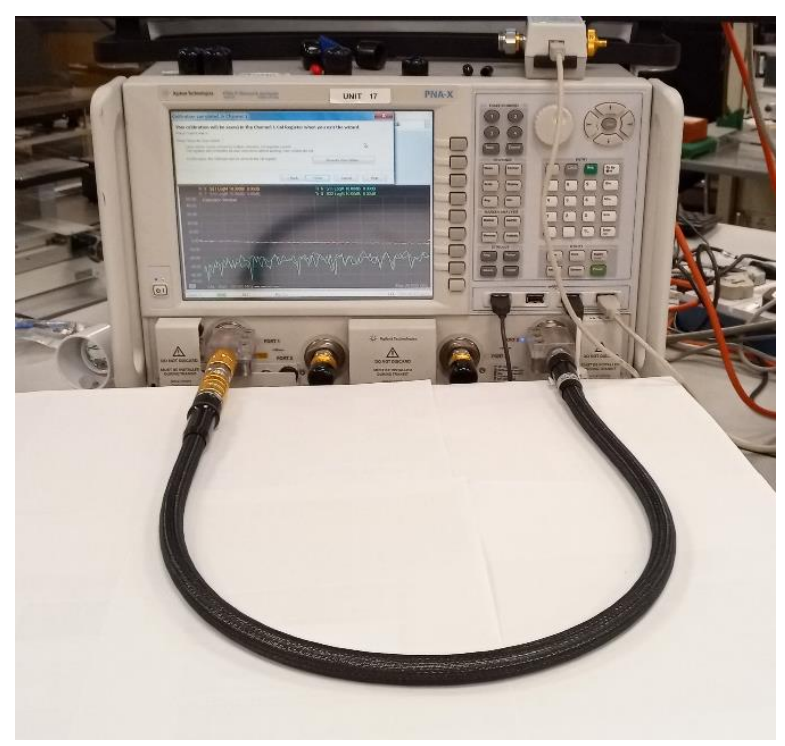

Figure 1: VNA measurement set-up

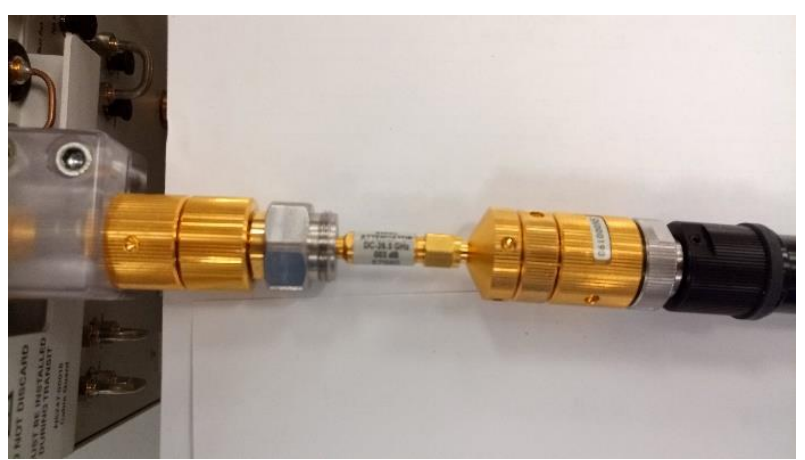

Figure 2: Two-port DUT connected to the VNA 


\section{VNA CALIBRATION METHODS}

In a VNA measurement set-up, components such as the measuring instrument, the cables and the connectors can introduce errors into the measurement. For example, impedance mismatches within the test setup cause errors that appear as ripples superimposed on the measured transmission and reflection coefficients. These systematic errors make it difficult to determine the actual S-parameters of the DUT as reflections from the DUT and from these error sources get mixed together. To estimate the systematic errors a calibration of the setup is done prior to the measurement of the DUT. After calibration, the systematic errors are de-embedded from the DUT measurements.

The following sub-sections give a brief overview of the VNA calibration methods investigated in this study without going into too much detail. Apart from PIMMS TRL, all the calibration methods investigated relied on the VNA firmware to perform the calibration and correct the DUT measurements. Other VNA calibration methods include relative calibrations, reflect response (using either a short or an open), transmission response and QSOLT. These are all low accuracy calibrations and are usually used for quick instrument setup where the user may be interested in only a subset of the two-port S-parameters. None of these calibration methods formed part of the investigation reported in this work.

\subsection{PIMMS TRL}

PIMMS (Primary Impedance Microwave Measurement System) [1] is NPL's primary standard measurement system for S-parameters at microwave frequencies. It is based on the use of precision coaxial air lines as impedance standards during a TRL calibration. Systematic errors in the air lines and in the VNA are assessed in separate experiments. Several repeat calibration and measurement runs are performed to assess the random errors in the measurements. In this work, for each repeat measurement of the DUTs, a single PIMMS calibration was used to perform a TRL calibration of the VNA and to correct the DUT measurements without using the VNA firmware.

\subsection{FIRMWARE TRL}

Thru-Reflect-Line (TRL) [3] is a very accurate calibration method. However, very few calibration kits contain TRL standards. TRL is a "self-calibration" technique which means that the calibration standards do not need to be fully characterised. The calibration algorithm itself estimates certain parameters of the line and the reflect standards. The characteristic impedance of the line standard sets the reference impedance for the measured S-parameters. The reflect standard is usually a short-circuit or an open-circuit and, for coaxial measurements, the line standard is an unsupported air dielectric coaxial line. The number of lines required depends on the frequency range since each line standard is only useable over an approximately 8:1 frequency range. The line length usually limits the lowest frequency at which TRL can be used since the long lines which would be required at low frequency are not practical to use.

In this investigation, three TRL line standards were used to cover the frequency range $100 \mathrm{MHz}$ to $26 \mathrm{GHz}$. The same calibration standards were used for both the PIMMS TRL and firmware TRL calibrations. These are listed in Table 1. 
Table 1: Calibration standards used for PIMMS TRL and firmware TRL calibrations

\begin{tabular}{|l|l|}
\hline Calibration standard & Description \\
\hline Zero-length Thru & Two VNA test ports connected together \\
\hline Reflect (short-circuit) & $\begin{array}{l}\text { Two short-circuits (one male and one female) } \\
\text { with assumed identical 5.25 mm offset. }\end{array}$ \\
\hline Line \#1 (frequency range 0.1 to $1.5 \mathrm{GHz}$ ) & $74.93 \mathrm{~mm}$ long coaxial air line \\
\hline Line \#2 (frequency range 1.5 to $7.0 \mathrm{GHz}$ ) & $16.17 \mathrm{~mm}$ long coaxial air line \\
\hline Line \#3 (frequency range 7.0 to $26 \mathrm{GHz}$ ) & $3.90 \mathrm{~mm}$ long coaxial air line \\
\hline
\end{tabular}

\subsection{SOLT}

Short-Open-Load-Thru (SOLT) [4] is a calibration method often used in industry to calibrate VNAs and can achieve high accuracy measurements. SOLT does not suffer from frequency bandwidth restrictions like TRL. Unlike TRL, the calibration standards for SOLT need to be fully characterised; the accuracy of the measured S-parameters of the DUT depends on the accuracy of the characterisation of the calibration standards. The manufacturer usually provides calibration standard definitions which can be used by the VNA.

The calibration standards used to perform the SOLT calibration in this investigation were taken from a $3.5 \mathrm{~mm}$ calibration kit, Model $8050 \mathrm{C}$, from Maury Microwave Corp. ${ }^{1}$ The thru used for the SOLT calibration was a zero length (flush) thru.

\section{$3.4 \quad$ SOLR}

Short-Open-Load-Reciprocal (SOLR), also known as the "unknown thru" calibration method [5], is like SOLT except that the S-parameters of the thru standard do not need to be known. The only assumption is that the thru is reciprocal, i.e. $S_{12}=S_{21}$. Like TRL, SOLR is a "self-calibration" method. This calibration works well provided the thru line is well matched $\left(S_{11}\right.$ and $S_{22}<-10 \mathrm{~dB}$ ) and low loss (>- $20 \mathrm{~dB})$.

In this investigation, two SOLR calibrations were performed: SOLR-0 mm with a zero length (flush) thru and SOLR-Reci with a non-zero length (non-flush) thru (approximately $15 \mathrm{~mm}$ line length). A $3.5 \mathrm{~mm}$ calibration kit, Model 8050C, from Maury Microwave Corp was used for the SOLR calibrations. ${ }^{1}$

\subsection{ECAL}

Most VNA vendors offer Electronic Calibration (ECal) modules [6] to make the calibration process fast and easy. The ECal has all the required terminations embedded in the module and these can be switched in sequence to provide highly repeatable reflection states. The ECal module is directly controlled by the VNA firmware through a USB cable and requires a single connection to the test ports to calibrate the test setup.

\footnotetext{
${ }^{1}$ This kit has NPL reference number CIS/C/306.
} 
In this investigation, a Keysight ECal module N4691D-60004 ${ }^{2}$ was used. Photographs of the ECal are shown in Figure 3. For the calibration used in this work, the internal ECal terminations were used together with an external flush thru to give a higher calibration accuracy.
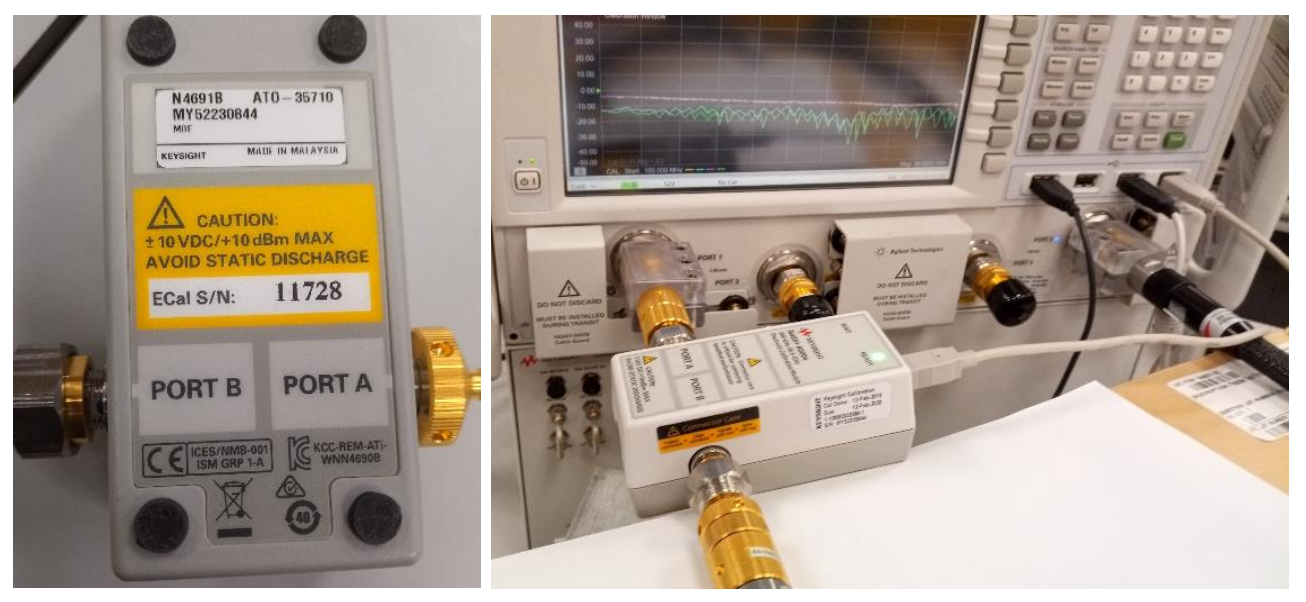

Figure 3: Photograph showing the ECal module used in this investigation

\section{DEVICES UNDER TEST}

The one-port and two-port DUTs measured in this investigation are listed in Table 2 and 3, respectively. The DUTs were chosen to provide a wide range of reflection and transmission coefficient values. Typical reflection and transmission performance of the DUTs are plotted in Figure 4 and Figure 55, respectively.

Table 2: One-port DUTs measured in this investigation

\begin{tabular}{|c|c|c|}
\hline One-port DUTs & Model Number & Description \\
\hline Offset Open-Circuit \#1 & MMC-8048A1 & Open $(\mathrm{F})$ with a $5.25 \mathrm{~mm}$ offset line \\
\hline Offset Open-Circuit \#2 & MMC-8048B1 & Open (M) with a $22.75 \mathrm{~mm}$ offset line \\
\hline Offset Short-Circuit \#1 & MMC-8046F & Short $(\mathrm{F})$ with a $5 \mathrm{~mm}$ offset line \\
\hline Offset Short-Circuit \#2 & MMC-8047F & Short (M) with $40 \mathrm{~mm}$ offset line \\
\hline 50 Ohm Load & MMC-8031A5 & $50 \Omega \operatorname{Load}(\mathrm{M})$ \\
\hline $\begin{array}{l}\text { Reflection } \\
\text { Check Standard \#1 }\end{array}$ & NPL-35F1 & NPL $3.5 \mathrm{~mm}(\mathrm{~F})$ reflection check standard \\
\hline $\begin{array}{l}\text { Reflection } \\
\text { Check Standard \#2 }\end{array}$ & NPL-35M1 & NPL $3.5 \mathrm{~mm}(\mathrm{M})$ reflection check standard \\
\hline
\end{tabular}

\footnotetext{
2 This ECal has Serial No 11728.
} 
Table 3: Two-port DUTs measured in this investigation

\begin{tabular}{|l|l|l|}
\hline Two-port DUTs & Part Number & Description \\
\hline Thru & N/A & Direct connection of two VNA ports \\
\hline Beatty Line & HP-85053-60004 & Coaxial Beatty Line \\
\hline $3 \mathrm{~dB}$ Attenuator & Agilent $-8493 \mathrm{C}$ & Attenuator \\
\hline $20 \mathrm{~dB}$ Attenuator & HP-85053-60001 & Attenuator \\
\hline $40 \mathrm{~dB}$ Attenuator & HP-85053-60002 & Attenuator \\
\hline
\end{tabular}

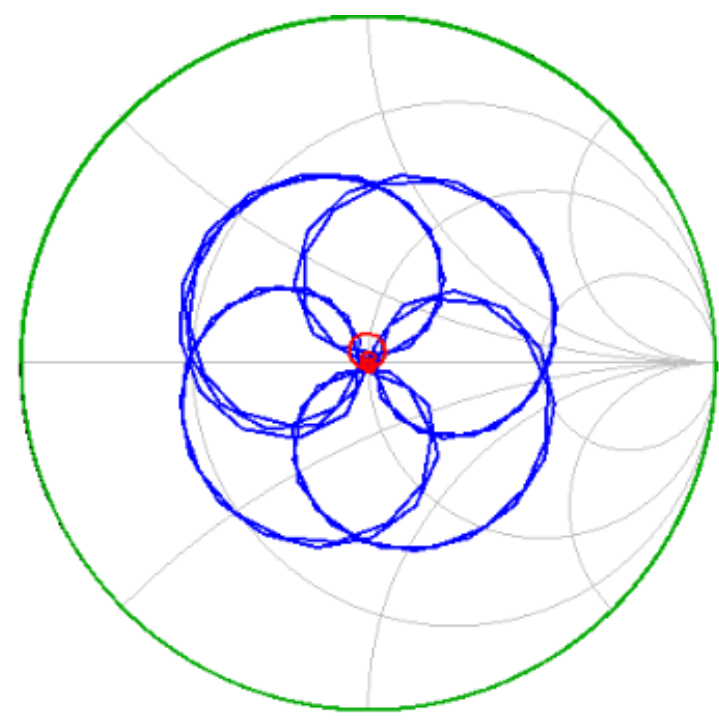

Figure 4: Typical reflection coefficients of one-port and two-port DUTs plotted from $100 \mathrm{MHz}$ to $26 \mathrm{GHz}$ on a Smith chart. The DUTs are: green - offset short-circuit; blue - Beatty line; red - Reflection Check Standard.

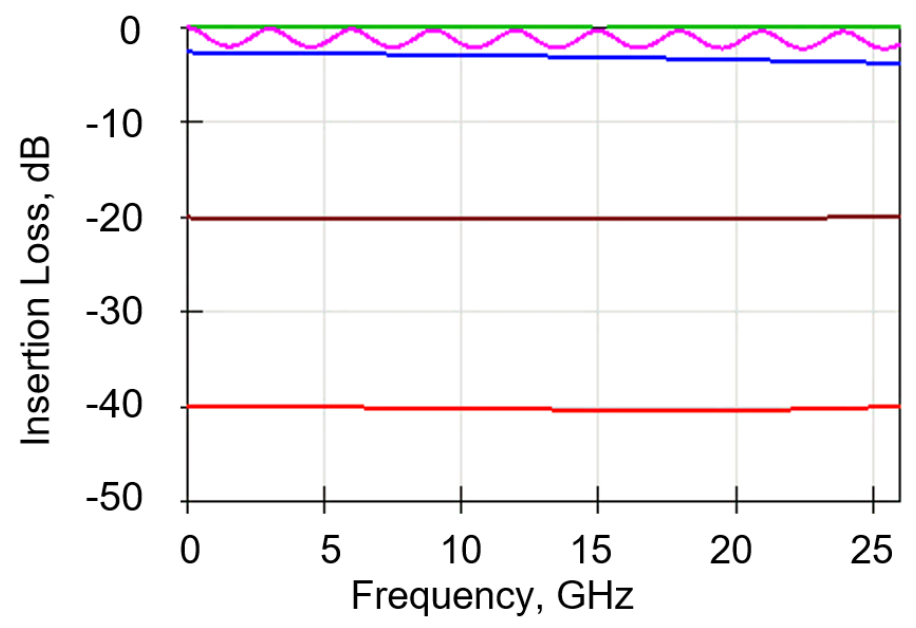

Figure 5: Typical insertion loss of two-port DUTs plotted from $100 \mathrm{MHz}$ to $26 \mathrm{GHz}$. The DUTs are: green - thru; pink - Beatty line; blue - $3 \mathrm{~dB}$ attenuator; brown - $20 \mathrm{~dB}$ attenuator; red - $40 \mathrm{~dB}$ attenuator. 


\section{DATA ANALYSIS}

The experimental standard deviation [7] was used to quantify the variability in the measurement results. The measured S-parameters are complex-valued quantities and so the standard deviation was computed separately for the magnitude and phase component of each S-parameter.

Let $S$ be a measured complex-valued S-parameter with $|S|$ and $\theta$ being the magnitude and phase components of $S$, respectively. $S$ can be written as follows:

$$
S=|S| \angle \theta
$$

For $n$ repeat measurements of $S, S_{k}=\left|S_{k}\right| \angle \theta_{k}$ for $k=1$ to $n$, the mean magnitude of $S$ can be computed as follows:

$$
\overline{|S|}=\frac{1}{n} \sum_{k=1}^{n}\left|S_{k}\right|
$$

and the experimental standard deviation in the magnitude of $S$ can be expressed as follows:

$$
s(|S|)=\sqrt{\frac{1}{n-1} \sum_{k=1}^{n}\left(\left|S_{k}\right|-\overline{|S|}\right)^{2}}
$$

Similarly, the mean phase of $S$ can be computed as follows:

$$
\bar{\theta}=\frac{1}{n} \sum_{k=1}^{n} \theta_{k}
$$

and the experimental standard deviation in the phase of $S$ can be expressed as follows:

$$
s(\theta)=\sqrt{\frac{1}{n-1} \sum_{k=1}^{n}\left(\theta_{k}-\bar{\theta}\right)^{2}}
$$

The phase is unwrapped before the mean and experimental standard deviation are calculated.

In our case, since each DUT is measured six times at each frequency, $n=6$. The values of $\overline{|S|}, s(|S|), \bar{\theta}$ and $s(\theta)$ are computed at each frequency using the six repeat measurements of each DUT from Equations (2) to (5).

\section{MEASUREMENT RESULTS}

For each calibration method, the following procedure was followed:

(i) the VNA was calibrated and the calibration coefficients (error terms) were saved to the VNA firmware;

(ii) each DUT was then connected to the VNA, in turn, and its S-parameters were measured;

(iii) steps (i) and (ii) were repeated 6 times to assess the variability due to the connection repeatability of the calibration standards, the DUTs and the cable. 
Female DUT ports were connected directly to Port 1 of the VNA whilst male DUT ports were connected to Port 2 of the VNA by means of a phase stable cable. The measurements were carried out with minimum movement in the cable to reduce the influence of cable non-repeatability.

For each calibration method and each DUT S-parameter, the mean magnitude $\overline{|S|}$, and the mean phase, $\bar{\theta}$, were calculated, at each frequency, using equations (2) and (4), respectively. Similarly, the experimental standard deviation in magnitude, $s(|S|)$, and the experimental standard deviation in phase, $s(\theta)$, were calculated, at each frequency, using equations (3) and (5), respectively.

Each measurement method was compared against PIMMS TRL (taken as a reference) by evaluating, for each DUT S-parameter, the difference in mean magnitude and the difference in mean phase for the S-parameter between the measurement method and PIMMS TRL as follows

$$
\begin{aligned}
& (\Delta|\bar{S}|)_{i}=|\bar{S}|_{i}-|\bar{S}|_{\text {PIMMS }} \\
& (\Delta \bar{\phi})_{i}=\bar{\phi}_{i}-\bar{\phi}_{\text {PIMMS }}
\end{aligned}
$$

where $|\bar{S}|_{i}$ is the mean magnitude of the S-parameter for method $i$ and $|\bar{S}|_{\text {PIMMS }}$ is the mean magnitude for PIMMS TRL and, similarly, $\bar{\phi}_{i}$ is the mean phase of the S-parameter for method $i$ and $\bar{\phi}_{\text {PIMMS }}$ is the mean phase for PIMMS TRL.

The means and experimental standard deviations for the magnitude and phase of the measured reflection and transmission coefficients as well as the differences from the PIMMS TRL measurement are plotted in Figure 6 to 12 for the one-port DUTs and in Figure 13 to 22 for the two-port DUTs. For S-parameters of small magnitude, corresponding to points close to the origin in the complex plane, the phase is not well defined. A small perturbation in the value of the S-parameter can result in a very large change in the value of its phase. For this reason, phase is not considered for S-parameters of small magnitude e.g. for the reflection coefficient of a well-matched device such as a $50 \Omega$ load or a precision attenuator.

\subsection{ONE-PORT DEVICES}

Seven one-port devices providing a range of reflection coefficient values were measured (see Table 2). The measured results for the one-port devices are presented in the following sub-sections.

\subsubsection{Offset Open-Circuits}

Two open-circuits (one male and one female) were measured during this investigation; open-circuit \#1 was a standard open-circuit with a $5.25 \mathrm{~mm}$ offset whilst open-circuit \#2 had an additional adaptor to provide extra phase length, totalling an offset of $22.75 \mathrm{~mm}$. The mean, difference relative to the PIMMS TRL measurements and experimental standard deviation of the magnitude and phase of reflection coefficient $\left(S_{11}\right)$ for the two open-circuits are plotted in Figure 6 and 7 for all the calibration methods.

The results show that the magnitude measured using all calibrations is within $10 \mathrm{mU}$, for most of the frequency range, and phase is within $1.5^{\circ}$ from the reference PIMMS results. Open-circuit results using the ECal and the SOLR-Zero calibration have larger ripple in the magnitude measurements than the other calibrations. The results show good agreement between the calibration methods. The experimental standard deviation for all calibration methods is less than $1.5 \mathrm{mU}$ for the magnitude and less than $0.1^{\circ}$ for the phase. 

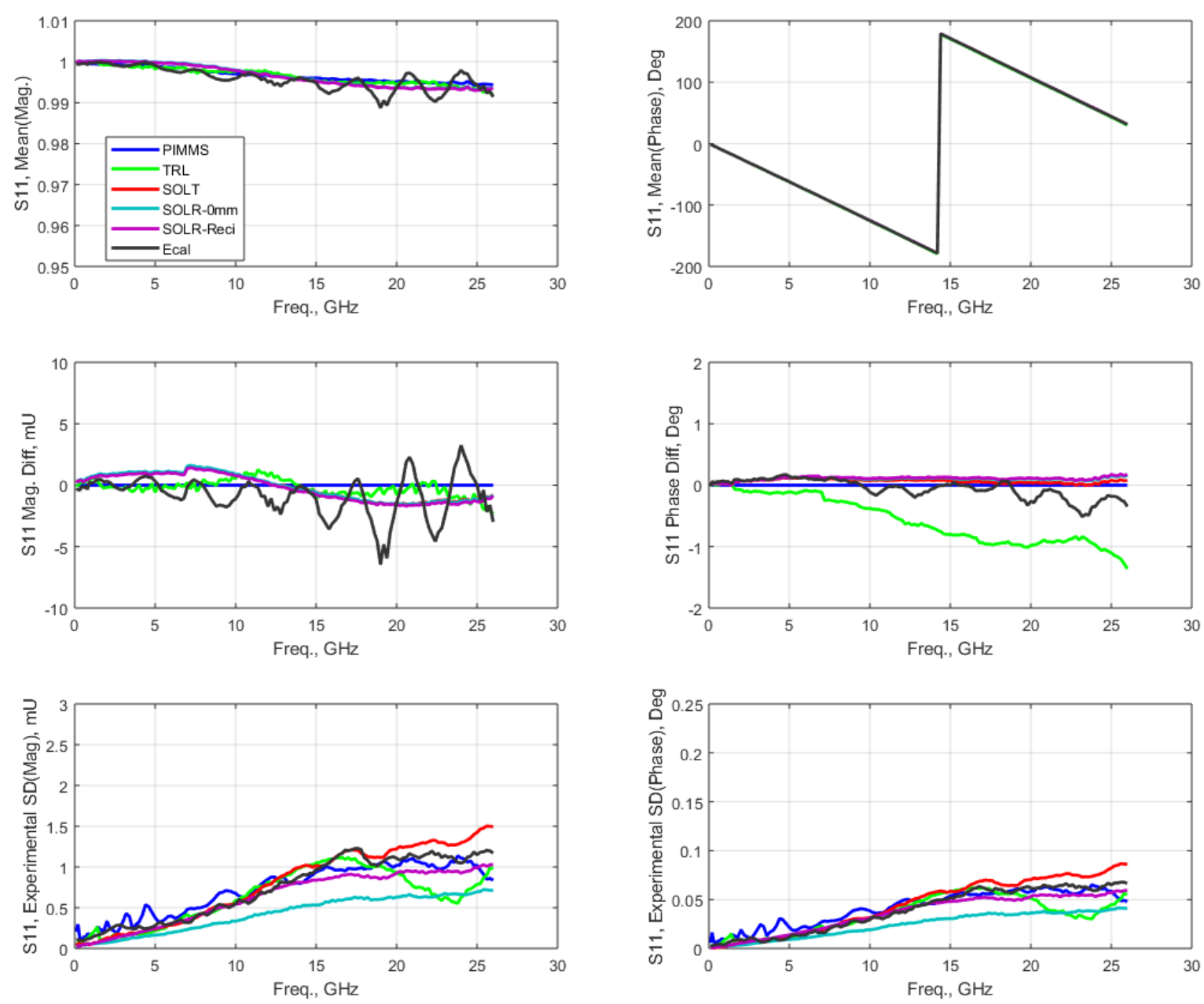

Figure 6: Mean, difference from PIMMS TRL and experimental standard deviation for $S_{11}$ of offset open-circuit \#1. 

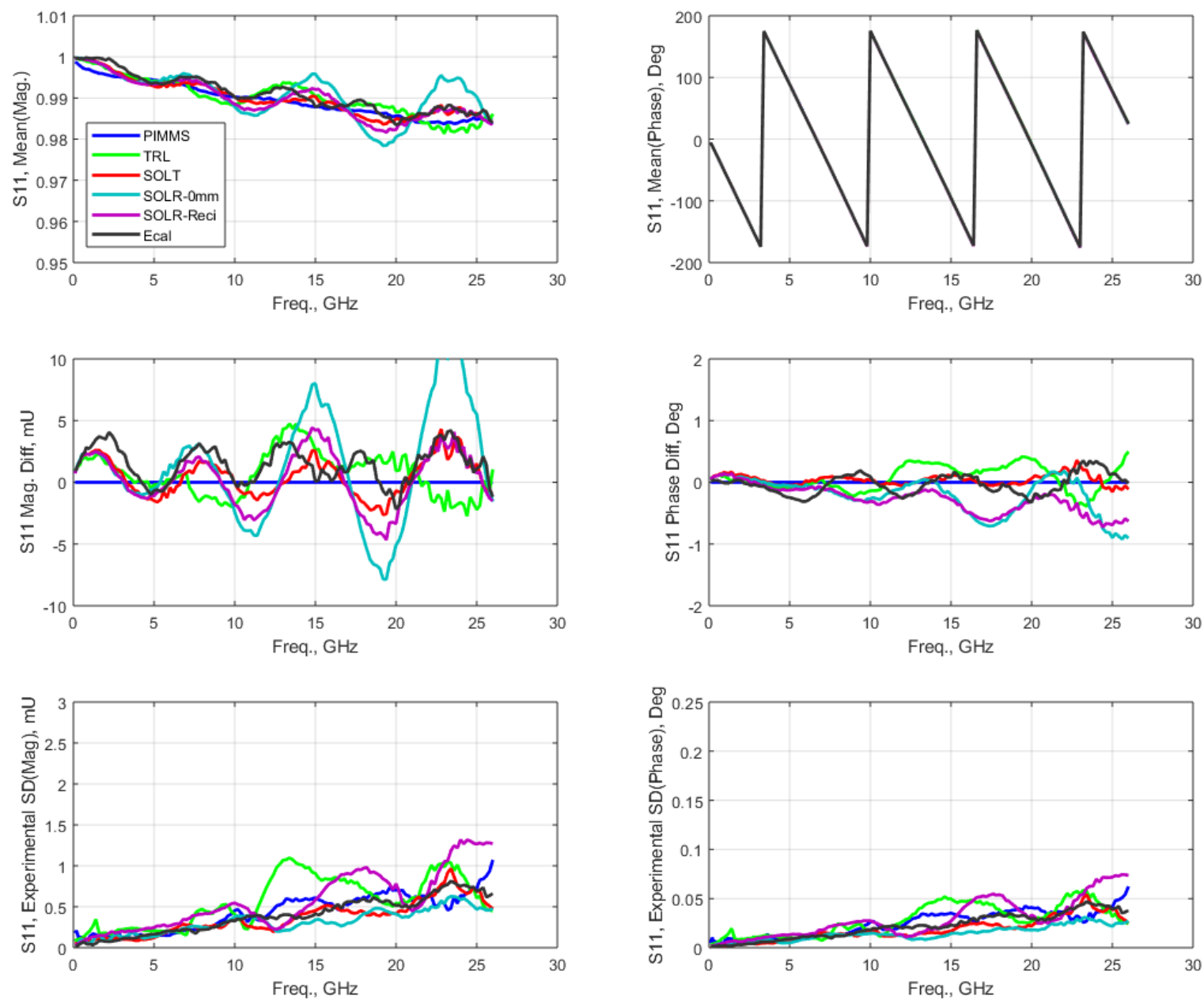

Figure 7: Mean, difference from PIMMS TRL and experimental standard deviation for $S_{11}$ of offset open-circuit \#2.

\subsubsection{Offset Short-Circuits}

Two short-circuits (one male and one female) were measured during this investigation; short-circuit \#1 was a $5 \mathrm{~mm}$ offset short-circuit whilst short-circuit \#2 had an additional adaptor to provide extra phase length, totalling an offset of $40 \mathrm{~mm}$. The mean, difference relative to the PIMMS TRL measurements and experimental standard deviation of the magnitude and phase of reflection coefficient $\left(\mathrm{S}_{11}\right)$ for the two short-circuits are plotted in Figure 8 and 9 for all the calibration methods.

The results show that the magnitude measured using all calibrations is within $10 \mathrm{mU}$ for most of the frequency range and phase is within $1^{\circ}$ from the reference PIMMS results. Short-circuit results using TRL, ECal and SOLR-Zero calibrations have larger ripples in the magnitude measurements than the other calibrations.

The results show good agreement between the calibration methods. The experimental standard deviation for all calibration methods is less than $2 \mathrm{mU}$ for magnitude and less than $0.1^{\circ}$ for the phase. 

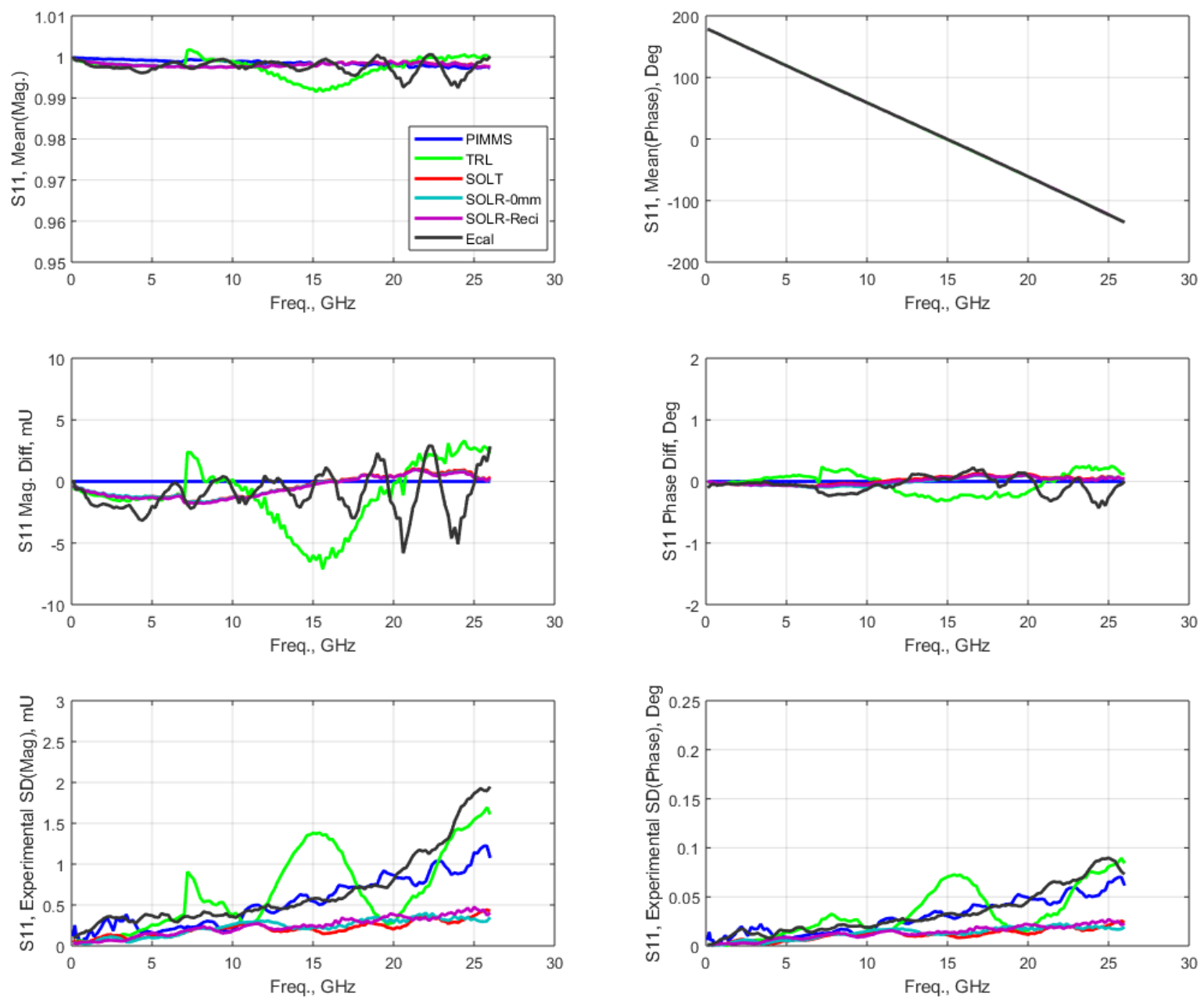

Figure 8: Mean, difference from PIMMS TRL and experimental standard deviation for $\mathrm{S}_{11}$ of offset short-circuit \#1. 

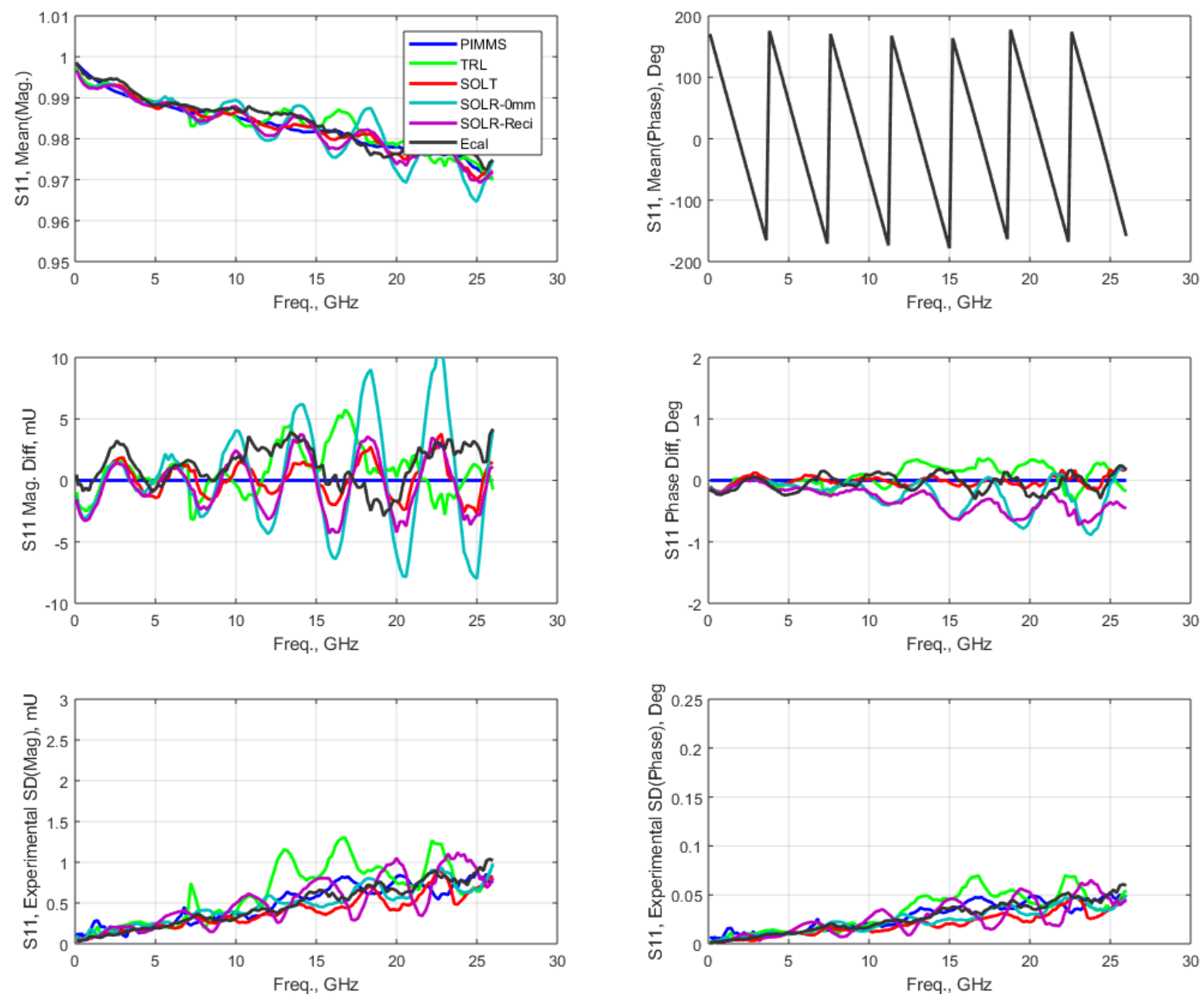

Figure 9: Mean, difference from PIMMS TRL and experimental standard deviation for $S_{11}$ of offset short-circuit \#2.

\subsection{3 $50 \mathrm{Ohm}$ Load}

A $50 \Omega$ load (male) was measured during the investigation. The mean, difference relative to the PIMMS TRL measurements and experimental standard deviation of the magnitude of reflection coefficient for the $50 \Omega$ load are plotted in Figure 10 for all the calibration methods. Note that only magnitude results for the load are shown as reflection coefficient phase for a very low reflect DUT can vary widely, due to the error encompassing the centre of the Smith chart. The PIMMS $S_{11}$ magnitude values at low frequencies are affected by a frequency-dependent systematic error due to a change in the characteristic impedance of the TRL calibration line standard at these frequencies. PIMMS usually corrects for this systematic error by applying an impedance renormalisation. However, on this occasion, this impedance renormalisation has not been applied.

The relative difference for magnitude between calibrations is less than $5 \mathrm{mU}$ for most of the frequency range. All calibration methods show similar deviation from the reference PIMMS measurement results. The results show good agreement between the calibration methods. The experimental standard deviation for all calibration methods is less than $0.4 \mathrm{mU}$ for the magnitude. 

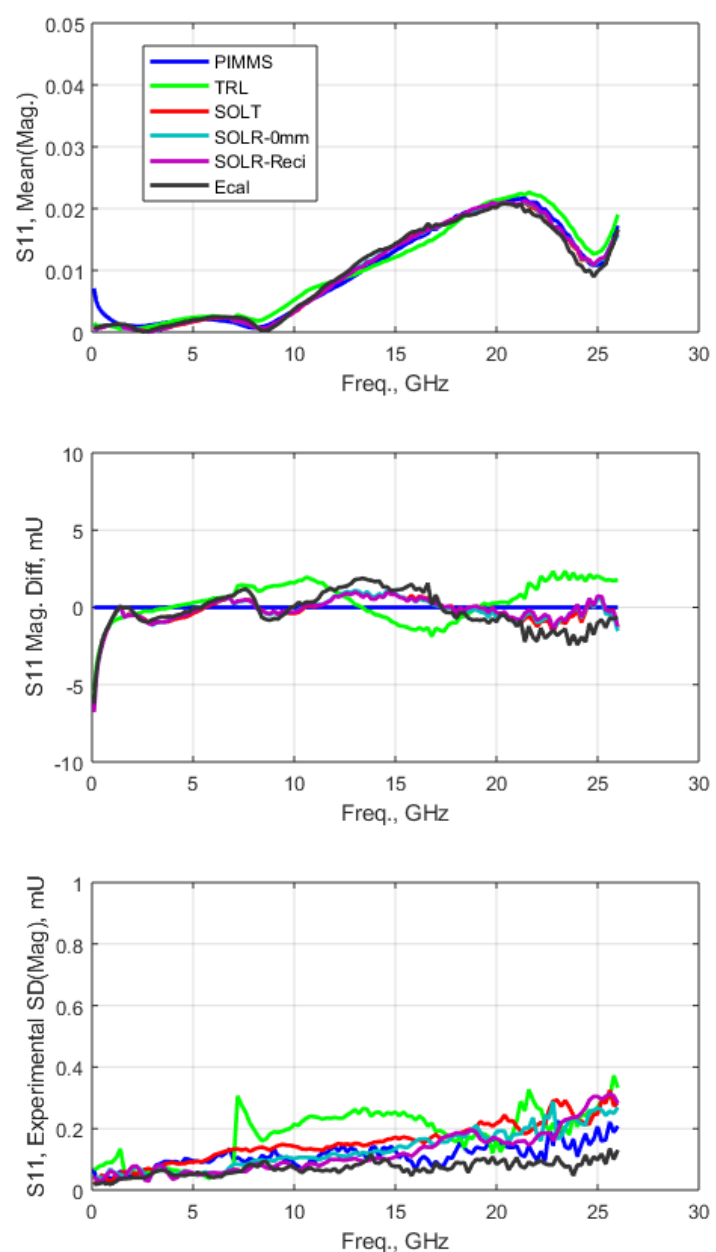

Figure 10: Mean, difference from PIMMS TRL and experimental standard deviation for S11 of $50 \Omega$ load.

\subsubsection{Reflection Check Standards}

Two one-port reflection check standards were measured during the investigation (one male and one female). These impedance standards, which are periodically characterised in terms of their reflection coefficients, are used at NPL to verify VNA reflection measurements. The mean, difference from PIMMS TRL and experimental standard deviation of the magnitude and phase of reflection coefficient for the two reflection check standards are plotted in Figure 11 and 12 for all the calibration methods.

The magnitude difference between all the calibrations and PIMMS TRL calibration is less than $7 \mathrm{mU}$ and for phase, it is less than $7^{\circ}$. SOLR and SOLT show greater difference in the results for the first check standard $(35 \mathrm{~F} 1)$.

The results show good agreement between the calibration methods. The experimental standard deviation for all calibration methods is less than $1 \mathrm{mU}$ for the magnitude and less than $0.5^{\circ}$ for the phase. Reflection check standard \#1 (35F1) shows slightly higher experimental standard deviation. 

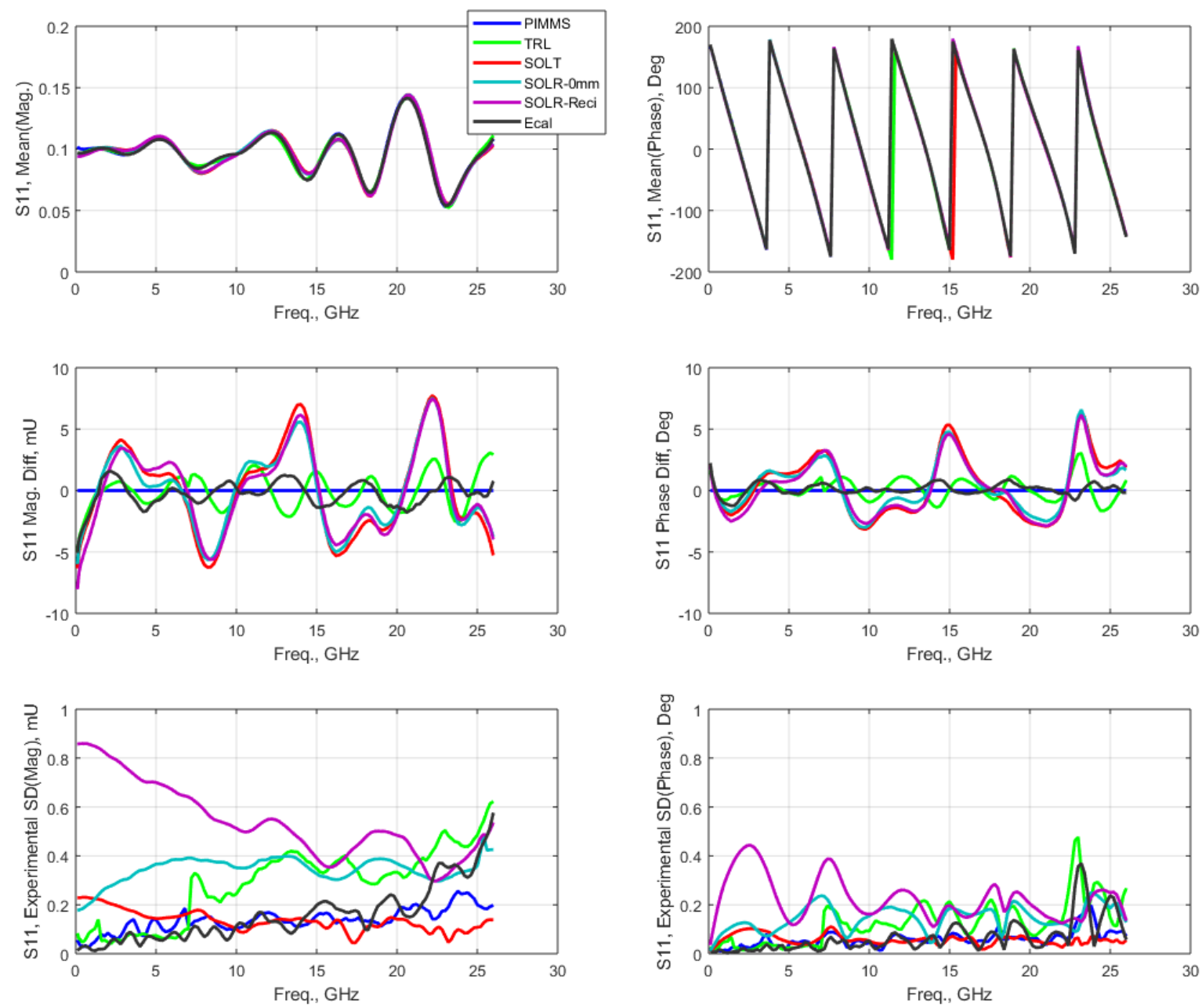

Figure 11: Mean, difference from PIMMS TRL and experimental standard deviation for $S_{11}$ of reflection check standard \#1. 

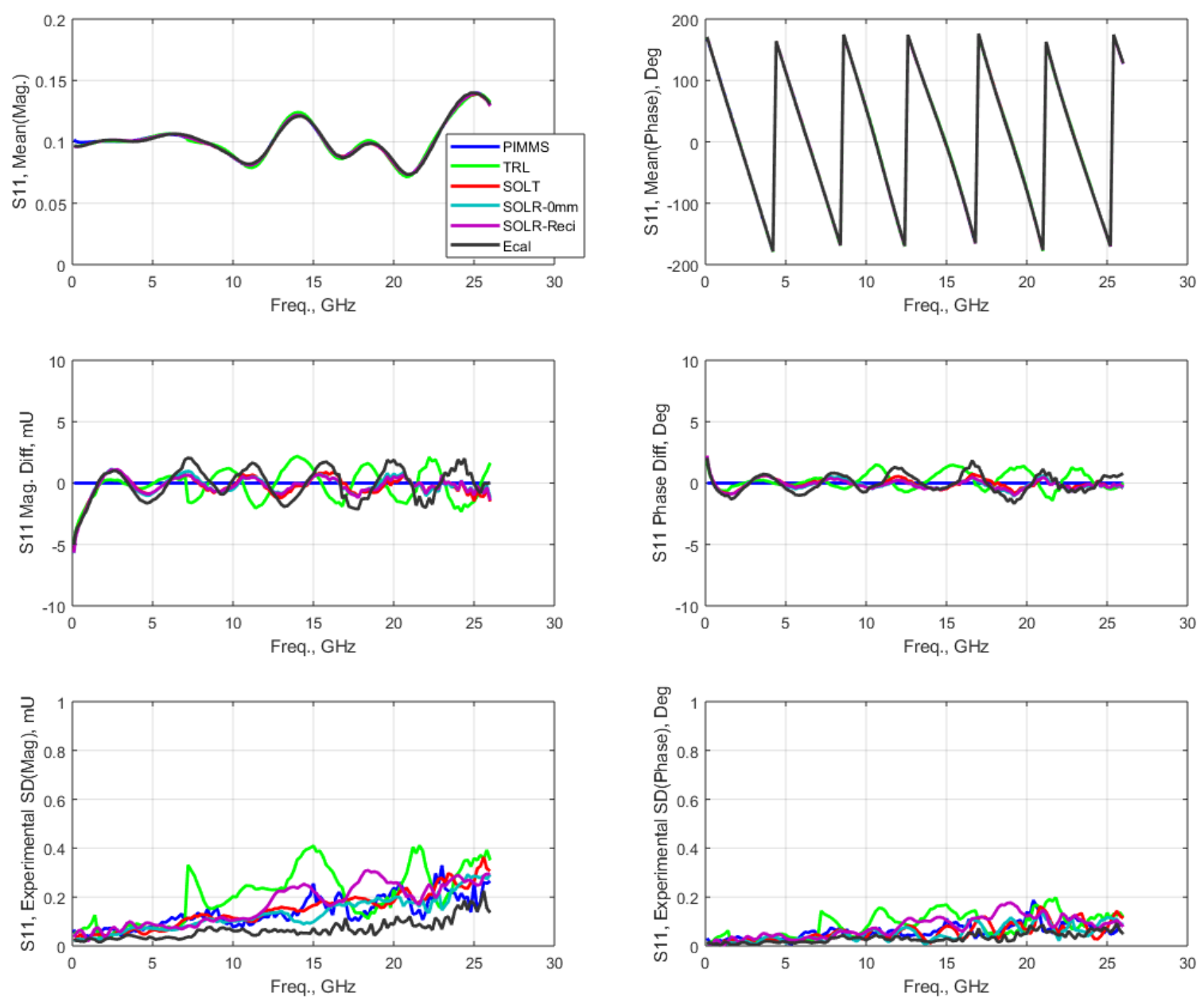

Figure 12: Mean, difference from PIMMS TRL and experimental standard deviation for $S_{11}$ of reflection check standard \#2.

\subsubsection{Summary of One-Port Devices}

The results for the one-port devices can be summarised by averaging the calibration differences and the experimental standard deviation over the measured frequency range. For all devices and all calibration methods, the averaged differences and the averaged experimental standard deviations of the reflection magnitude component are listed in Tables 4 and 5, respectively. For all devices and all calibration methods, the averaged differences and the averaged experimental standard deviations of the reflection phase are listed in Tables 6 and 7, respectively.

The following conclusions can be drawn from these tables:

1) The maximum averaged difference of all measurements is less than $3.5 \mathrm{mU}$ for magnitude and $2^{\circ}$ for phase.

2) The experimental standard deviation averaged over frequency is less than $0.8 \mathrm{mU}$ for magnitude and less than $0.15^{\circ}$ for phase.

3) The averaged experimental standard deviation for magnitude is lower for low reflect devices.

4) The averaged experimental standard deviation for phase is lower for high reflect devices.

5) When the results for all devices are analyzed, each calibration gives similar average values for the experimental standard deviation.

6) From all the results it can be seen that the experimental standard deviation for all calibrations is 
considerably smaller than the relative differences to reference PIMMS measurements.

Table 4: $\mathrm{S}_{11}$ magnitude difference $(\mathrm{mU})$ relative to PIMMS averaged over frequency for each one-port device and calibration method.

\begin{tabular}{|l|c|c|c|c|c|c|}
\hline DUT & PIMMS & \multicolumn{1}{l|}{ TRL } & SOLT & SOLR - 0 mm & SOLR-Reci & ECAL \\
\hline Open \#1 & 0.00 & 0.53 & 0.95 & 0.99 & 0.99 & 1.27 \\
\hline Open \#2 & 0.00 & 1.54 & 1.33 & 3.52 & 1.87 & 1.72 \\
\hline Short \#1 & 0.00 & 2.14 & 0.82 & 0.80 & 0.84 & 1.50 \\
\hline Short \#2 & 0.00 & 1.53 & 1.30 & 3.35 & 1.88 & 1.66 \\
\hline Load & 0.00 & 1.53 & 1.30 & 3.35 & 1.88 & 1.66 \\
\hline 35F1 & 0.00 & 1.24 & 3.12 & 2.73 & 2.95 & 0.87 \\
\hline 35M1 & 0.00 & 1.11 & 0.74 & 0.72 & 0.68 & 1.16 \\
\hline
\end{tabular}

Table 5: Experimental standard deviation in $S_{11}$ magnitude $(\mathrm{mU})$ averaged over frequency for each oneport device and calibration method.

\begin{tabular}{|l|c|c|c|c|c|c|}
\hline DUT & PIMMS & \multicolumn{1}{l|}{ TRL } & SOLT & SOLR - 0 mm & SOLR-Reci & ECAL \\
\hline Open \#1 & 0.71 & 0.62 & 0.76 & 0.41 & 0.61 & 0.72 \\
\hline Open \#2 & 0.42 & 0.55 & 0.35 & 0.30 & 0.55 & 0.38 \\
\hline Short \#1 & 0.51 & 0.64 & 0.21 & 0.22 & 0.23 & 0.65 \\
\hline Short \#2 & 0.47 & 0.61 & 0.38 & 0.46 & 0.50 & 0.46 \\
\hline Load & 0.11 & 0.18 & 0.15 & 0.13 & 0.12 & 0.07 \\
\hline 35F1 & 0.13 & 0.28 & 0.14 & 0.34 & 0.55 & 0.15 \\
\hline 35M1 & 0.13 & 0.20 & 0.14 & 0.13 & 0.16 & 0.07 \\
\hline
\end{tabular}

Table 6: $S_{11}$ phase $\left(^{\circ}\right)$ difference relative to PIMMS averaged over frequency for each one-port device and calibration method.

\begin{tabular}{|l|c|c|c|c|c|c|}
\hline DUT & PIMMS & TRL & SOLT & SOLR - 0 mm & SOLR-Reci & ECAL \\
\hline Open \#1 & 0.00 & 0.56 & 0.07 & 0.10 & 0.11 & 0.12 \\
\hline Open \#2 & 0.00 & 0.17 & 0.08 & 0.24 & 0.29 & 0.13 \\
\hline Short \#1 & 0.00 & 0.14 & 0.06 & 0.05 & 0.05 & 0.11 \\
\hline Short \#2 & 0.00 & 0.15 & 0.07 & 0.24 & 0.31 & 0.12 \\
\hline Load & - & - & - & - & - & - \\
\hline 35F1 & 0.00 & 0.65 & 2.01 & 1.70 & 1.80 & 0.37 \\
\hline 35M1 & 0.00 & 0.55 & 0.34 & 0.36 & 0.34 & 0.63 \\
\hline
\end{tabular}

Table 7: Experimental standard deviation in $S_{11}$ phase $\left(^{\circ}\right)$ averaged over frequency for each one-port device and calibration method.

\begin{tabular}{|l|c|c|c|c|c|c|}
\hline DUT & PIMMS & TRL & SOLT & SOLR - 0 mm & SOLR-Reci & ECAL \\
\hline Open \#1 & 0.04 & 0.03 & 0.04 & 0.02 & 0.03 & 0.04 \\
\hline Open \#2 & 0.02 & 0.03 & 0.02 & 0.01 & 0.03 & 0.02 \\
\hline Short \#1 & 0.03 & 0.03 & 0.01 & 0.01 & 0.01 & 0.03 \\
\hline Short \#2 & 0.03 & 0.03 & 0.02 & 0.02 & 0.03 & 0.03 \\
\hline Load & - & - & - & - & - & - \\
\hline 35F1 & 0.06 & 0.12 & 0.06 & 0.14 & 0.22 & 0.06 \\
\hline 35M1 & 0.06 & 0.08 & 0.06 & 0.05 & 0.08 & 0.03 \\
\hline
\end{tabular}




\subsection{TWO-PORT DEVICES}

Five two-port devices providing a range of reflection and transmission coefficient values were measured (see Table 3). Only the transmission coefficients from port 1 to port $2\left(\mathrm{~S}_{21}\right)$ are plotted for the two-port DUTs as $S_{12}$ results are expected to be very similar to $S_{21}$. Similarly, only reflection coefficients from port $1\left(\mathrm{~S}_{11}\right)$ are plotted in this report. The measured results for the two-port devices are presented in the following sub-sections.

\subsubsection{Thru}

The Thru measurements were carried out by connecting measurement port 1 directly to measurement port 2 . The Thru measurements were made immediately after all calibrations.

The mean, difference relative to the PIMMS TRL measurements and experimental standard deviation of the magnitude and phase of reflection coefficient $\left(S_{11}\right)$ and transmission coefficient $\left(S_{21}\right)$ for the Thru are plotted in Figures 13 and 14, respectively, for all the calibration methods. The phase of $S_{11}$ for the Thru is not plotted since the Thru is well matched i.e. the magnitude of its reflection coefficient is small.

The results from the two SOLR calibration methods are significantly different to those from the other calibrations. The results from the ECal, SOLT and TRL calibrations show good agreement with the PIMMS results. The maximum SOLR $S_{11}$ magnitude difference is up to $15 \mathrm{mU}$. The experimental standard deviation is also higher for the SOLR calibration results. For all the calibration methods, the reflection coefficients $\left(S_{11}\right)$ show an experimental standard deviation less than $2.5 \mathrm{mU}$ in magnitude. The phase of $S_{11}$ is not plotted as it is considered irrelevant for very low reflect measurements. For all the calibration methods, the transmission coefficients $\left(S_{21}\right)$ show an experimental standard deviation less than $1 \mathrm{mU}$ in magnitude and less than $0.06^{\circ}$ in phase. 
NPL Report TQE 14
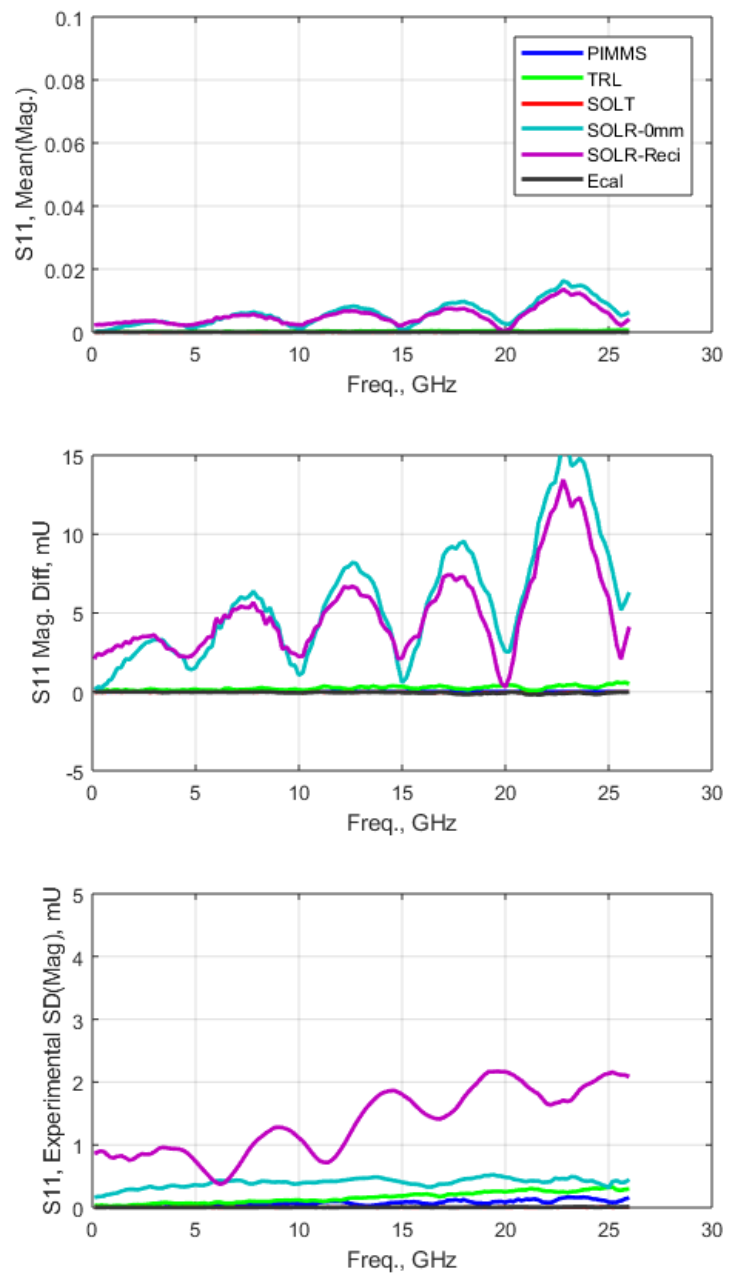

Figure 13: Mean, difference from PIMMS TRL and experimental standard deviation for magnitude of $S_{11}$ of Thru. 

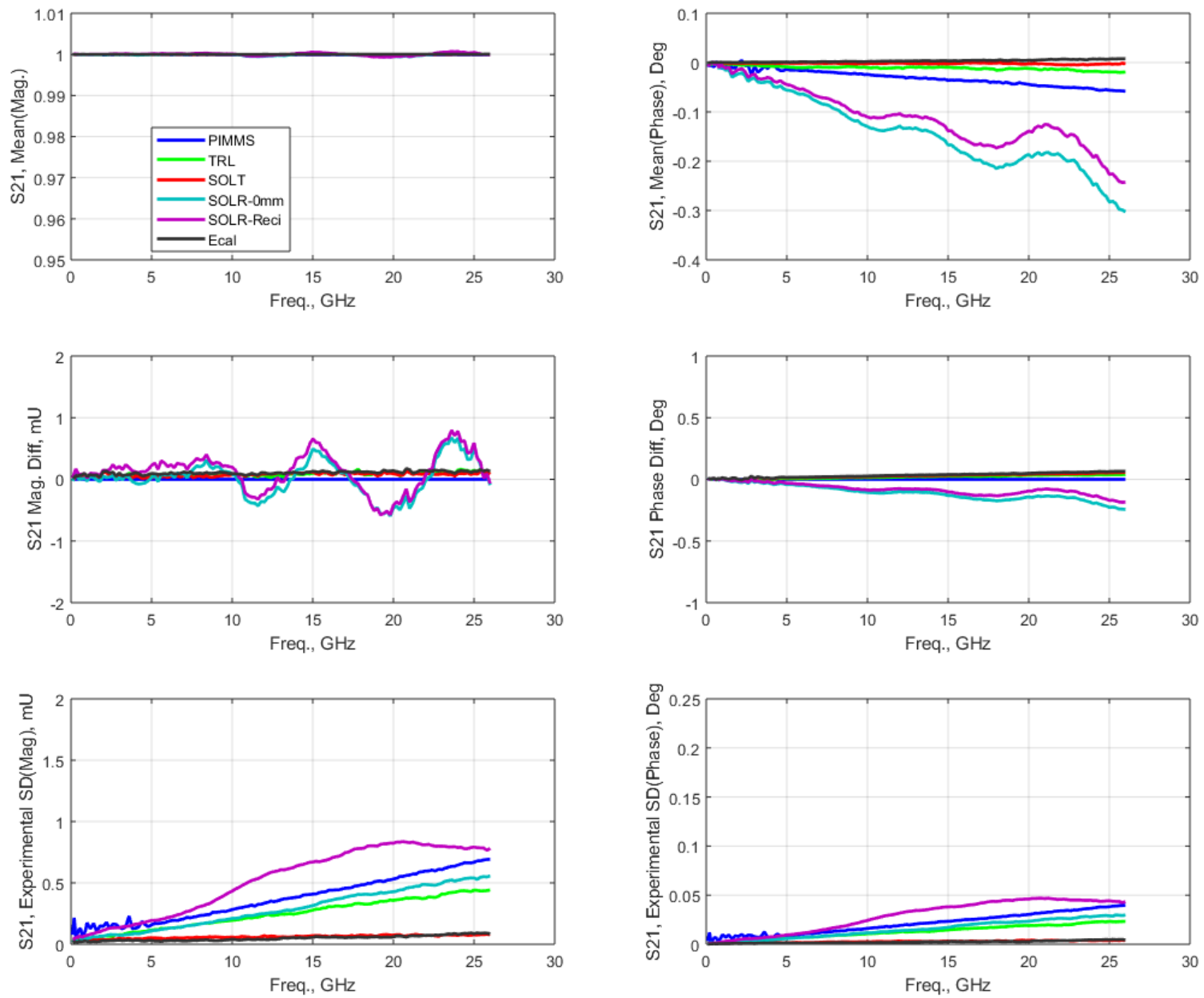

Figure 14: Mean, difference from PIMMS TRL and experimental standard deviation for $S_{21}$ of Thru.

\subsubsection{Beatty Line}

A coaxial line with a stepped characteristic impedance going from $50 \Omega$ to $25 \Omega$ and back to $50 \Omega$, also known as a Beatty line [8], was measured as one of the two-port DUTs. A Beatty line is a highly resonant structure and provides a wide range of reflection and transmission coefficients across the frequency range, which is very useful in microwave metrology.

The mean, difference relative to the PIMMS TRL measurements and experimental standard deviation of the magnitude and phase of reflection coefficient $\left(S_{11}\right)$ and transmission coefficient $\left(S_{21}\right)$ for the Beatty line are plotted in Figures 15 and 16 for all the calibration methods.

Figure 15 shows results for $S_{11}$ of the Beatty line. The difference in $S_{11}$ between PIMMS TRL and the other calibration methods can be more than $10 \mathrm{mU}$ for the magnitude and more than $10^{\circ}$ for the phase. For all the calibration methods, the experimental standard deviation of $S_{11}$ magnitude is less than $8 \mathrm{mU}$. The experimental standard deviation in the phase of $S_{11}$ varies a lot with frequency as expected for a load of varying impedance. The experimental standard deviation in phase of $S_{11}$ shows peaks at 
frequencies where the reflection coefficient magnitude is small; a similar situation occurs for the oneport $50 \Omega$ load where the magnitude of $S_{11}$ is very small and the phase is very variable.

Figure 16 shows results for transmission coefficients $\left(S_{21}\right)$ of the Beatty line. The $S_{21}$ results show magnitude differences from PIMMS TRL less than $8 \mathrm{mU}$ and an experimental standard deviation less than $3 \mathrm{mU}$. The differences in $\mathrm{S}_{21}$ phase from PIMMS TRL are less than $0.5^{\circ}$ and the experimental standard deviation in the phase of $S_{21}$ is less than $0.2^{\circ}$.
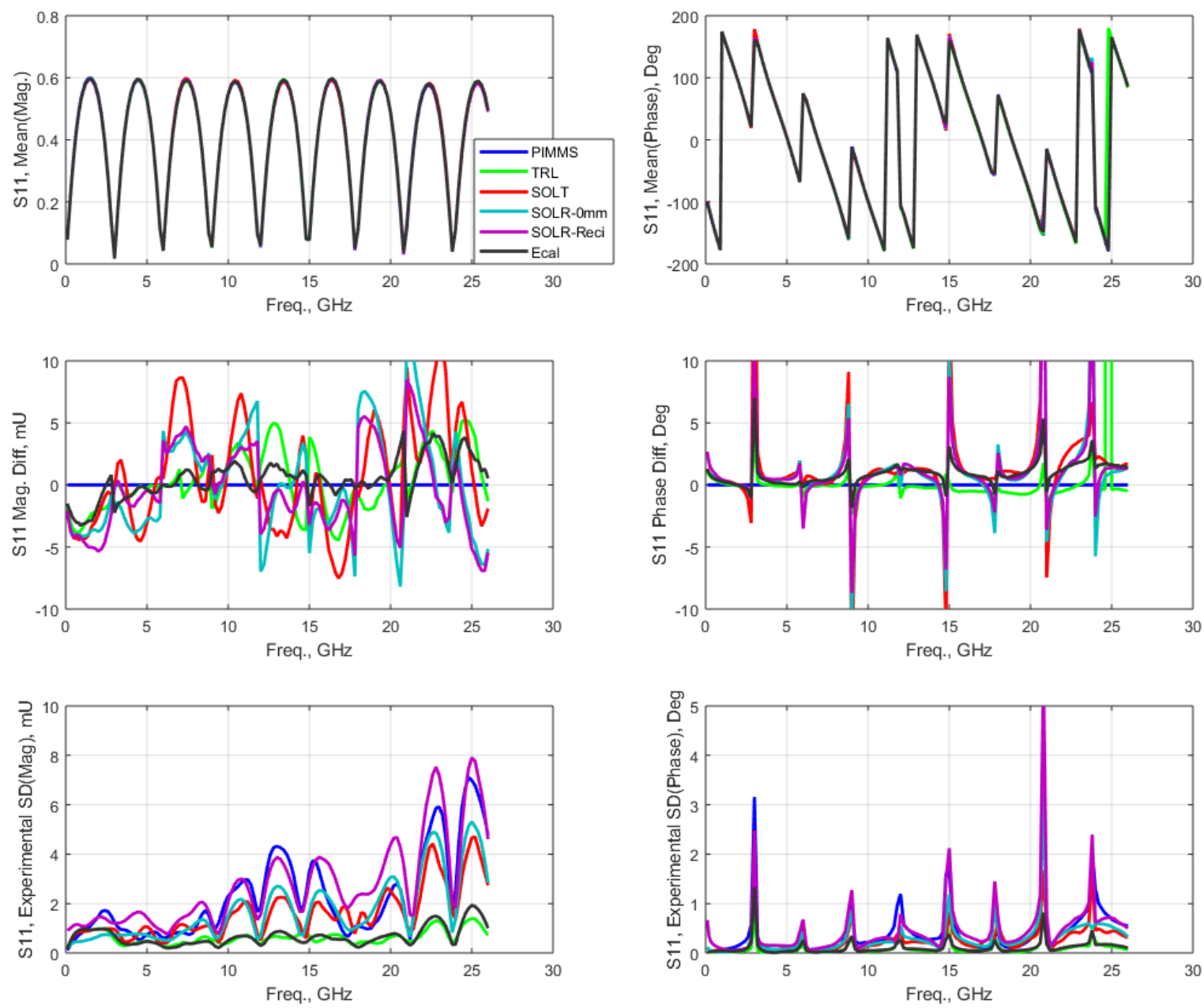

Figure 15: Mean, difference from PIMMS TRL and experimental standard deviation for $\mathrm{S}_{11}$ of Beatty line. 

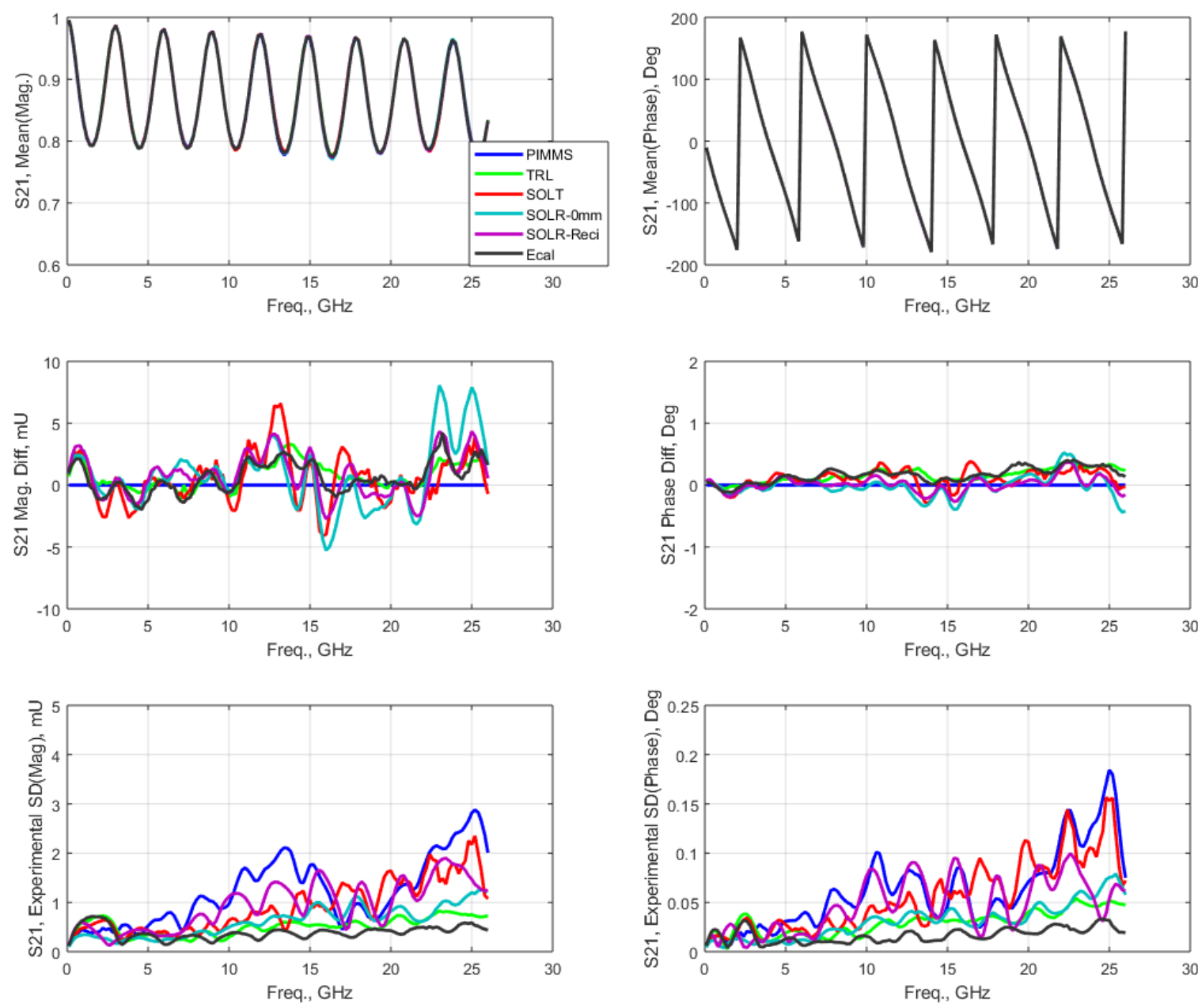

Figure 16: Mean, difference from PIMMS TRL and experimental standard deviation of S21 for Beatty line.

\subsection{3 $3 \mathrm{~dB}$ Attenuator}

The mean, difference from PIMMS TRL measurements and experimental standard deviation of the magnitude of $S_{11}$ of the $3 \mathrm{~dB}$ attenuator for the different calibration methods are plotted in Figure 17. The phase of $S_{11}$ for this and the other attenuators is not plotted because the phase for a very low reflect DUT can vary widely, due to the error encompassing the centre of the Smith chart. The maximum difference between PIMMS TRL and other calibration methods is less than $8 \mathrm{mU}$ for the magnitude of $\mathrm{S}_{11}$. The experimental standard deviation of $S_{11}$ is less than $1.2 \mathrm{mU}$ for the magnitude. 

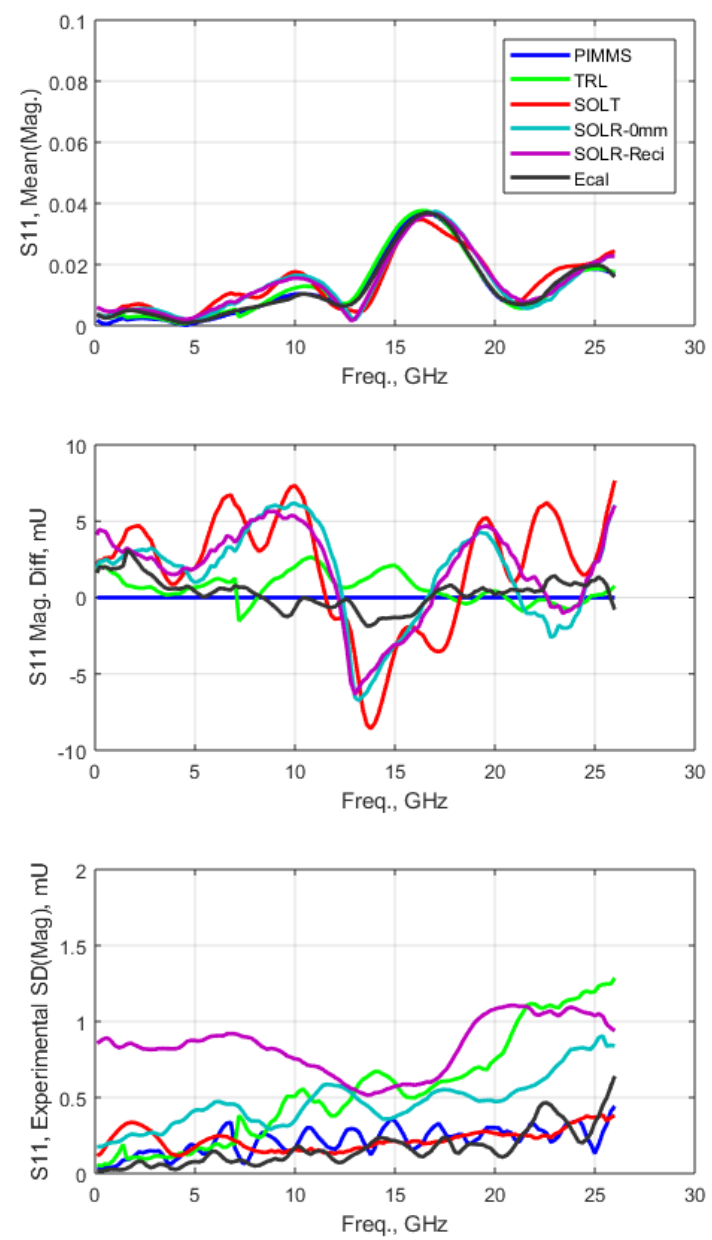

Figure 17: Mean, difference from PIMMS TRL and experimental standard deviation of magnitude of $S_{11}$ for $3 \mathrm{~dB}$ attenuator.

The mean, difference from PIMMS TRL and experimental standard deviation of the magnitude and phase of $S_{21}$ of the $3 \mathrm{~dB}$ attenuator for the different calibration methods are plotted in Figure 18. The maximum $S_{21}$ difference between PIMMS TRL and other calibration methods can be up to $2 \mathrm{mU}$ for the magnitude and $0.2^{\circ}$ for the phase. The $S_{21}$ values show an experimental standard deviation less than 1.2 $\mathrm{mU}$ in magnitude and less than $0.1^{\circ}$ in phase. 

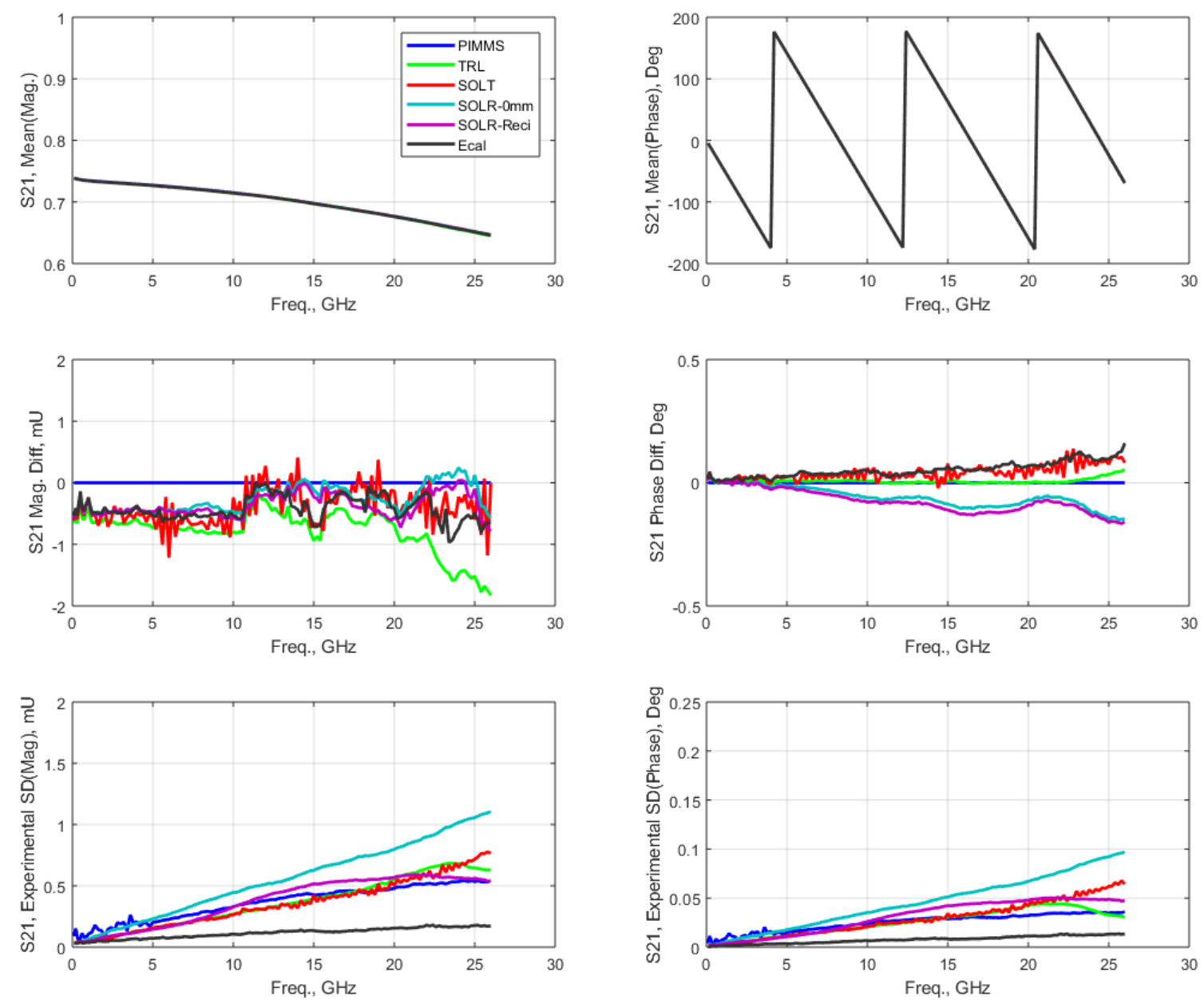

Figure 18: Mean, difference from PIMMS TRL and experimental standard deviation of $S_{21}$ for $3 \mathrm{~dB}$ attenuator.

\subsection{4 $20 \mathrm{~dB}$ Attenuator}

The mean, difference from PIMMS TRL measurements and experimental standard deviation of the magnitude of $S_{11}$ of the $20 \mathrm{~dB}$ attenuator for the different calibration methods are plotted in Figure 19. The maximum difference between PIMMS TRL and the other calibration results can be up to $8 \mathrm{mU}$ for magnitude of $S_{11}$. The experimental standard deviation of $S_{11}$ magnitude is less than $1.2 \mathrm{mU}$. 

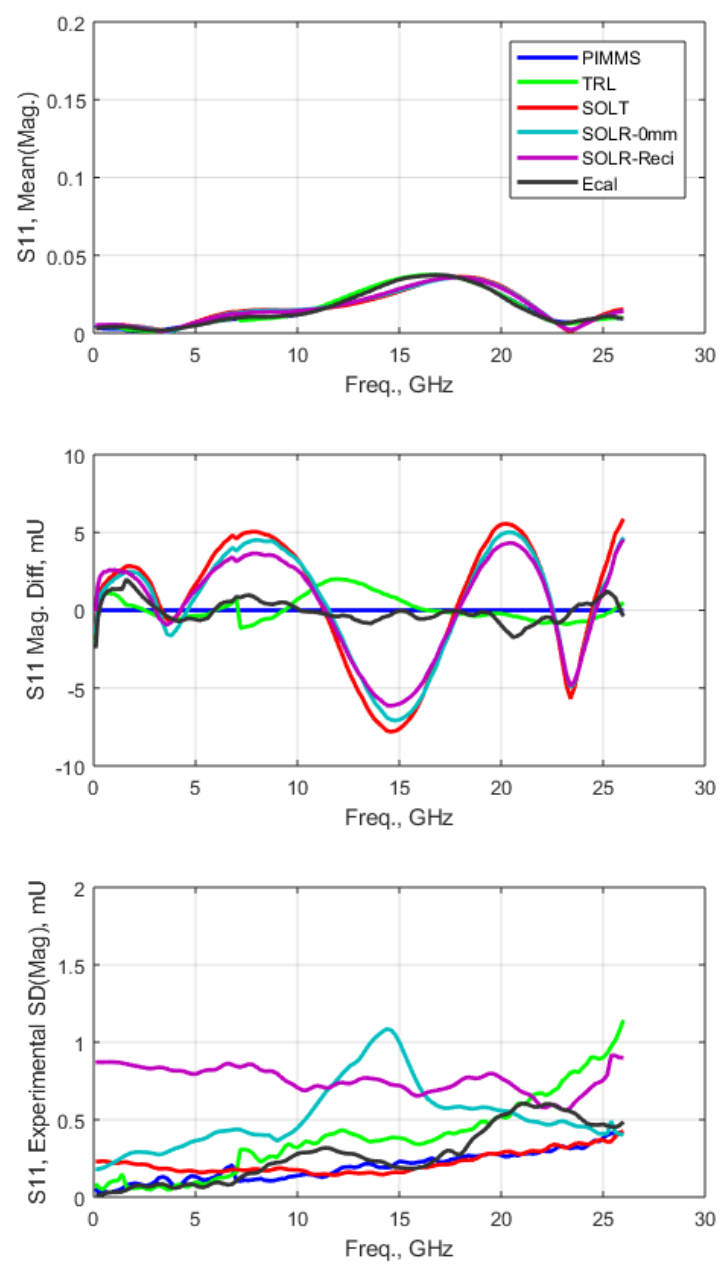

Figure 19: Mean, difference from PIMMS TRL and experimental standard deviation in magnitude of $S_{11}$ for $20 \mathrm{~dB}$ attenuator.

The mean, difference from PIMMS TRL and experimental standard deviation of the magnitude and phase of $S_{21}$ of the $20 \mathrm{~dB}$ attenuator for the different calibration methods are plotted in Figure 20. The maximum $S_{21}$ difference between PIMMS TRL and the other calibration results can be up to $1 \mathrm{mU}$ for magnitude and $1^{\circ}$ for phase. The $S_{21}$ values show an experimental standard deviation less than $0.6 \mathrm{mU}$ in magnitude and less than $0.4^{\circ}$ in phase for all the calibration methods. 

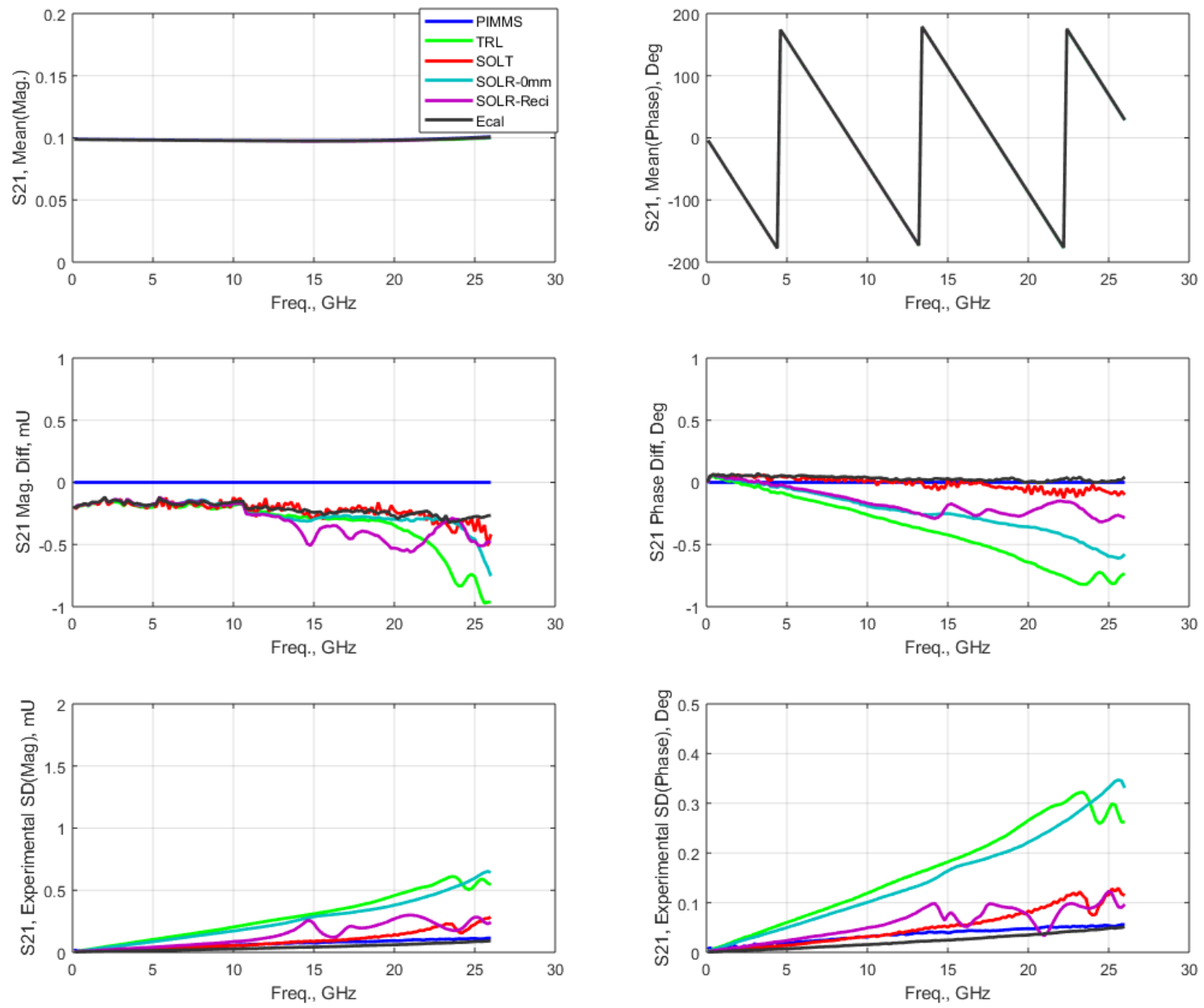

Figure 20: Mean, difference from PIMMS TRL and experimental standard deviation of $S_{21}$ for $20 \mathrm{~dB}$ attenuator.

\subsection{5 $40 \mathrm{~dB}$ Attenuator}

The mean, difference relative to the PIMMS TRL measurements and experimental standard deviation of the magnitude and phase of reflection coefficient $\left(S_{11}\right)$ and transmission coefficient $\left(S_{21}\right)$ for the $40 \mathrm{~dB}$ attenuator are plotted in Figure 21 and Figure 22 for all the calibration methods. The phase of $S_{11}$ for the $40 \mathrm{~dB}$ attenuator is not plotted since the device is well matched i.e. the magnitude of its reflection coefficient is small.

Figure 21 shows results for $S_{11}$ of the $40 \mathrm{~dB}$ attenuator. The maximum difference in magnitude of $S_{11}$ between PIMMS TRL and the other calibration methods is up to $8 \mathrm{mU}$ for magnitude. The experimental standard deviation of $S_{11}$ magnitude is less than $0.8 \mathrm{mU}$ for all the calibration methods.

Figure 22 shows results for $\mathrm{S}_{21}$ of the $40 \mathrm{~dB}$ attenuator. The maximum difference in $\mathrm{S}_{21}$ between PIMMS and the other calibration methods is up to $0.03 \mathrm{mU}$ for magnitude and $0.2^{\circ}$ for phase. The $\mathrm{S}_{21}$ values 
show an experimental standard deviation less than $0.02 \mathrm{mU}$ in magnitude and less than $0.07^{\circ}$ in phase for all the calibration methods.
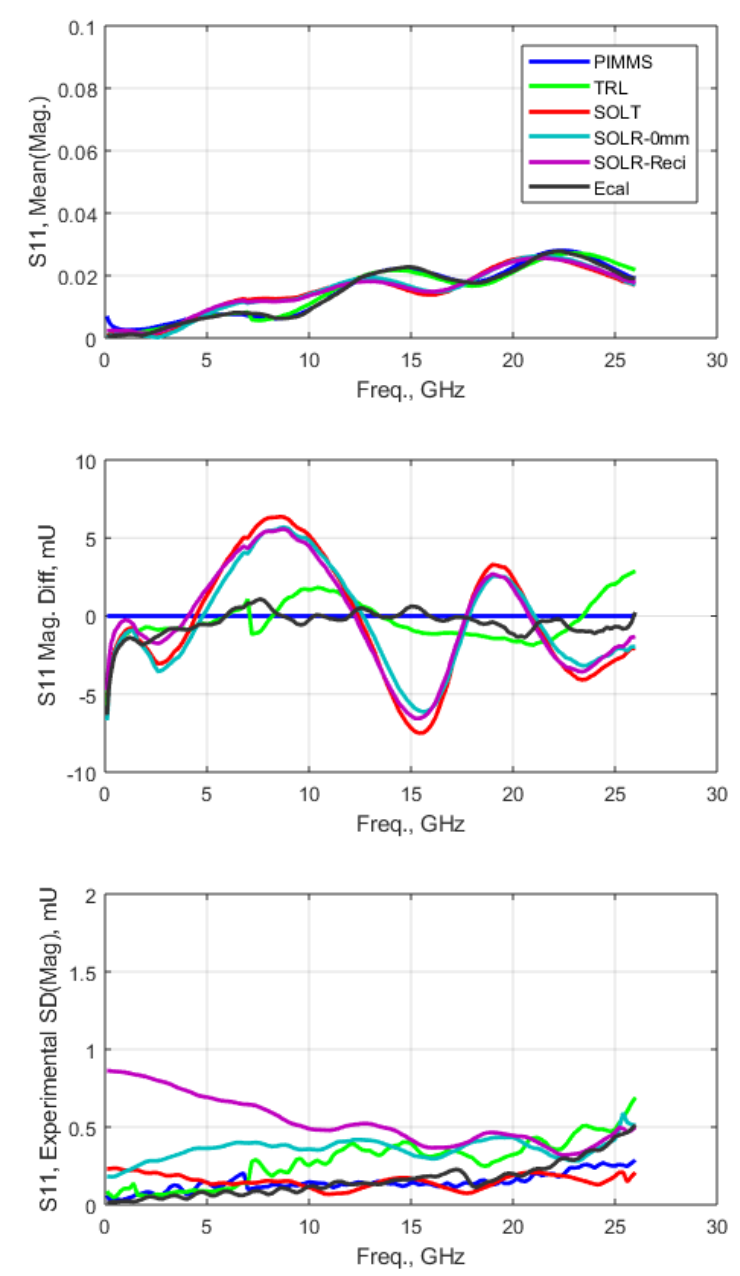

Figure 21: Mean, difference from PIMMS TRL and experimental standard deviation in magnitude of $S_{11}$ for $40 \mathrm{~dB}$ attenuator. 

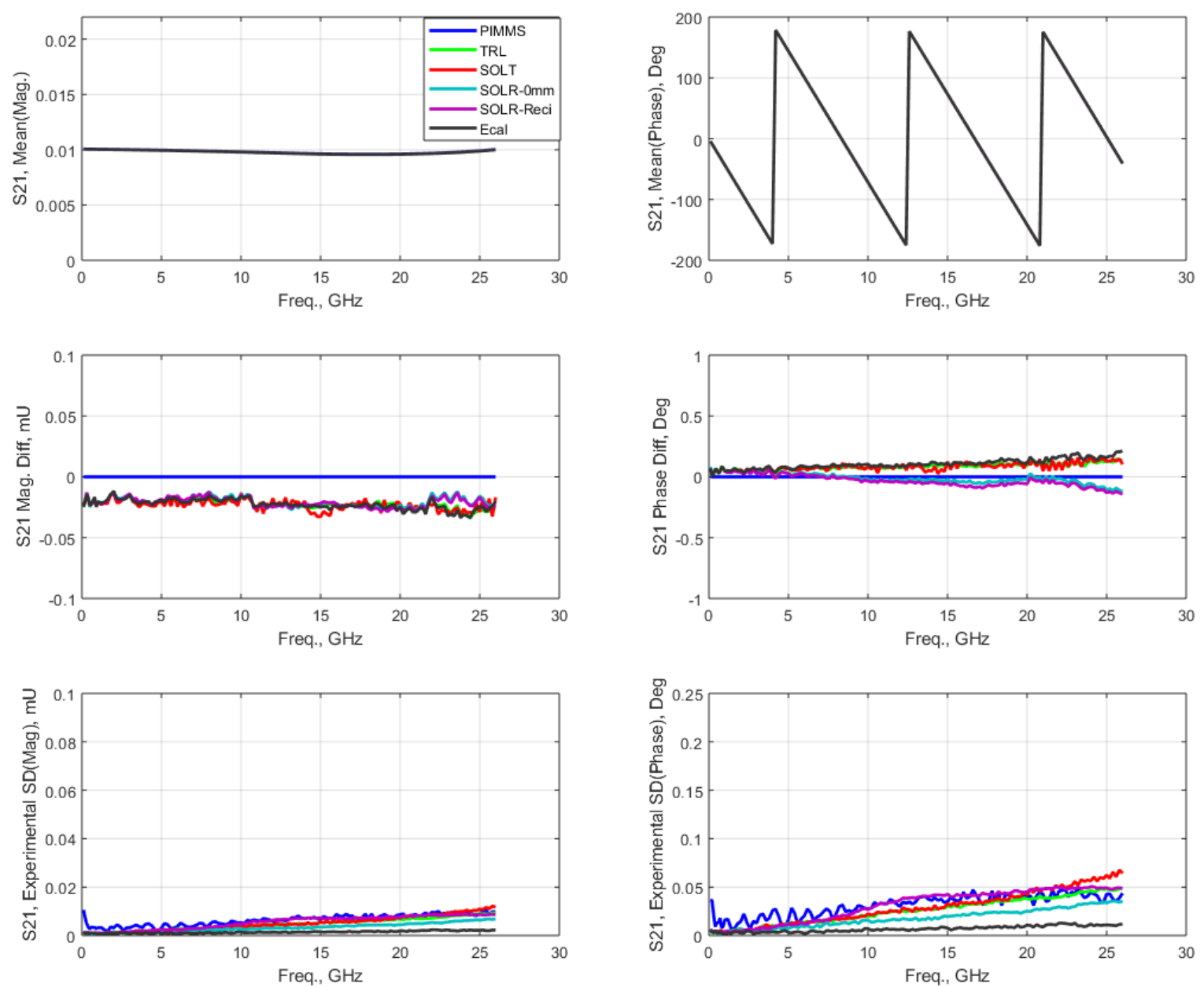

Figure 22: Mean, difference from PIMMS TRL and experimental standard deviation of $S_{21}$ for $40 \mathrm{~dB}$ attenuator.

\subsubsection{Summary of Two-Port Devices}

The results from the two-port devices can be summarised by averaging the experimental standard deviation over the measured frequency range. The differences from PIMMS TRL can also be averaged over the frequency range. For all two-port devices and all calibration methods, the averaged results are listed in tables as follows:

Table 8 - $\mathrm{S}_{11}$, Magnitude differences averaged over frequency

Table 9 - $\mathrm{S}_{11}$, Magnitude experimental standard deviation averaged over frequency

Table $10-\mathrm{S}_{11}$, Phase differences averaged over frequency

Table 11- $S_{11}$, Phase experimental standard deviation averaged over frequency

Table 12 - $\mathrm{S}_{21}$, Magnitude differences averaged over frequency

Table 13 - $S_{21}$, Magnitude experimental standard deviation averaged over frequency

Table $14-\mathrm{S}_{21}$, Phase differences averaged over frequency

Table $15-S_{21}$, Phase experimental standard deviation averaged over frequency

The following conclusions can be drawn from these results: 
1) The averaged differences from PIMMS TRL for $S_{11}$ magnitude are less than $5.6 \mathrm{mU}$ for all calibration methods; SOLT and SOLR show greater differences than FIRMWARE TRL and ECal.

2) The averaged experimental standard deviations in $S_{11}$ magnitude are less than $2.7 \mathrm{mU}$ for all calibration methods. Most calibrations show similar values with SOLR-Reciprocal being higher than the others.

3) The Beatty line is the only 2-port DUT for which reflection coefficient phase is compared. The averaged differences from PIMMS TRL for $S_{11}$ phase are less than $3.1^{\circ}$ and averaged experimental standard deviations of less than $0.45^{\circ}$ are seen.

4) The averaged differences from PIMMS TRL for $S_{21}$ magnitude are less than $2 \mathrm{mU}$ for all calibration methods.

5) The averaged experimental standard deviations for $S_{21}$ magnitude are less than $1.2 \mathrm{mU}$ for all calibration methods.

6) The averaged differences from PIMMS TRL for $S_{21}$ phase are less than $0.37^{\circ}$ for all calibration methods.

7) The averaged experimental standard deviations for $S_{21}$ phase are less than $0.16^{\circ}$ for all calibration methods.

8) The averaged differences from PIMMS TRL and averaged experimental standard deviations for DUTs with approximately constant impedance across the frequency range are lower than for DUTs whose impedance has a strong frequency dependence (Beatty line).

9) ECal shows lower averaged experimental standard deviations than the other calibration methods, this is mainly due to the smaller number of connections needed during the calibration process.

Table 8: $\mathrm{S}_{11}$ magnitude difference $(\mathrm{mU})$ relative to PIMMS averaged over frequency for each two-port device and calibration method.

\begin{tabular}{|l|c|c|c|c|c|c|}
\hline DUT & PIMMS & TRL & SOLT & SOLR-0 mm & SOLR-Reci & ECAL \\
\hline Thru & 0.00 & 0.23 & 0.05 & 5.53 & 4.92 & 0.05 \\
\hline Beatty & 0.00 & 1.98 & 3.57 & 3.43 & 2.89 & 1.28 \\
\hline 3 dB Attenuator & 0.00 & 0.91 & 3.70 & 3.04 & 3.07 & 0.87 \\
\hline 20 dB Attenuator & 0.00 & 0.66 & 3.40 & 3.00 & 2.71 & 0.60 \\
\hline 40 dB Attenuator & 0.00 & 1.18 & 3.27 & 2.85 & 2.74 & 0.74 \\
\hline
\end{tabular}

Table 9: Experimental standard deviation in $\mathrm{S}_{11}$ magnitude $(\mathrm{mU})$ averaged over frequency for each twoport device and calibration method.

\begin{tabular}{|l|c|c|c|c|c|c|}
\hline DUT & PIMMS & TRL & SOLT & SOLR-0 mm & SOLR-Reci & ECAL \\
\hline Thru & 0.07 & 0.16 & 0.01 & 0.39 & 1.35 & 0.01 \\
\hline Beatty & 2.21 & 0.63 & 1.56 & 1.70 & 2.69 & 0.67 \\
\hline 3 dB Attenuator & 0.21 & 0.53 & 0.22 & 0.46 & 0.84 & 0.16 \\
\hline 20 dB Attenuator & 0.19 & 0.38 & 0.22 & 0.51 & 0.76 & 0.27 \\
\hline 40 dB Attenuator & 0.14 & 0.28 & 0.16 & 0.35 & 0.55 & 0.15 \\
\hline
\end{tabular}

Table 10: $\mathrm{S}_{11}$ phase difference $\left(^{\circ}\right)$ relative to PIMMS averaged over frequency for each calibration method for the Beatty line.

\begin{tabular}{|l|c|c|c|c|c|c|}
\hline DUT & PIMMS & TRL & SOLT & SOLR-0 mm & SOLR-Reci & ECAL \\
\hline Beatty & 0.00 & 3.10 & 2.10 & 1.81 & 1.61 & 1.00 \\
\hline
\end{tabular}


Table 11: Experimental standard deviation in $S_{11}$ phase $\left(^{\circ}\right)$ averaged over frequency for each calibration method for the Beatty line.

\begin{tabular}{|l|c|c|c|c|c|c|}
\hline DUT & PIMMS & TRL & SOLT & SOLR-0 mm & SOLR-Reci & ECAL \\
\hline Beatty & 0.39 & 0.09 & 0.26 & 0.30 & 0.45 & 0.10 \\
\hline
\end{tabular}

Table 12: $S_{21}$ magnitude difference (mU) relative to PIMMS averaged over frequency for each two-port device and calibration method.

\begin{tabular}{|l|c|c|c|c|c|c|}
\hline DUT & PIMMS & TRL & SOLT & SOLR-0 mm & SOLR-Reci & ECAL \\
\hline Thru & 0.00 & 0.09 & 0.07 & 0.21 & 0.26 & 0.11 \\
\hline 3 dB Attenuator & 0.00 & 0.77 & 0.43 & 0.30 & 0.36 & 0.43 \\
\hline 20 dB Attenuator & 0.00 & 0.32 & 0.22 & 0.25 & 0.30 & 0.21 \\
\hline 40 dB Attenuator & 0.00 & 0.02 & 0.02 & 0.02 & 0.02 & 0.02 \\
\hline Beatty & 0.00 & 1.01 & 1.59 & 2.04 & 1.53 & 1.10 \\
\hline
\end{tabular}

Table 13: Experimental standard deviation in $S_{21}$ magnitude $(\mathrm{mU})$ averaged over frequency for each twoport device and calibration method.

\begin{tabular}{|l|c|c|c|c|c|c|}
\hline DUT & PIMMS & TRL & SOLT & SOLR-0 mm & SOLR-Reci & ECAL \\
\hline Thru & 0.36 & 0.24 & 0.06 & 0.28 & 0.51 & 0.05 \\
\hline 3 dB Attenuator & 0.35 & 0.35 & 0.34 & 0.54 & 0.37 & 0.11 \\
\hline 20 dB Attenuator & 0.07 & 0.28 & 0.09 & 0.25 & 0.13 & 0.04 \\
\hline 40 dB Attenuator & 0.01 & 0.00 & 0.00 & 0.00 & 0.01 & 0.00 \\
\hline Beatty & 1.17 & 0.51 & 0.89 & 0.60 & 0.92 & 0.39 \\
\hline
\end{tabular}

Table 14: $\mathrm{S}_{21}$ phase difference $\left(^{\circ}\right)$ relative to PIMMS averaged over frequency for each two-port device and calibration method.

\begin{tabular}{|l|c|c|c|c|c|c|}
\hline DUT & PIMMS & TRL & SOLT & SOLR-0 mm & SOLR-Reci & ECAL \\
\hline Thru & 0.00 & 0.02 & 0.03 & 0.11 & 0.08 & 0.03 \\
\hline 3 dB Attenuator & 0.00 & 0.01 & 0.04 & 0.06 & 0.07 & 0.05 \\
\hline 20 dB Attenuator & 0.00 & 0.37 & 0.04 & 0.24 & 0.16 & 0.03 \\
\hline 40 dB Attenuator & 0.00 & 0.08 & 0.08 & 0.03 & 0.05 & 0.11 \\
\hline Beatty & 0.00 & 0.15 & 0.14 & 0.12 & 0.08 & 0.17 \\
\hline
\end{tabular}

Table 15: Experimental standard deviation in $S_{21}$ phase $\left(^{\circ}\right)$ averaged over frequency for each two-port device and calibration method.

\begin{tabular}{|l|c|c|c|c|c|c|}
\hline DUT & PIMMS & TRL & SOLT & SOLR-0 mm & SOLR-Reci & ECAL \\
\hline Thru & 0.02 & 0.01 & 0.00 & 0.01 & 0.03 & 0.00 \\
\hline 3 dB Attenuator & 0.02 & 0.02 & 0.03 & 0.04 & 0.03 & 0.01 \\
\hline 20 dB Attenuator & 0.03 & 0.16 & 0.05 & 0.14 & 0.06 & 0.02 \\
\hline 40 dB Attenuator & 0.03 & 0.02 & 0.03 & 0.02 & 0.03 & 0.01 \\
\hline Beatty & 0.06 & 0.03 & 0.05 & 0.03 & 0.05 & 0.02 \\
\hline
\end{tabular}




\section{CONCLUSIONS}

Seven one-port DUTs were used in the calibration repeatability investigation: two offset short-circuits, two offset open-circuits, two reflection check standards and a $50 \Omega$ termination. Five two-port DUTs were used in the investigation: a thru, a Beatty line, a $3 \mathrm{~dB}$ attenuator, a $20 \mathrm{~dB}$ attenuator and a $40 \mathrm{~dB}$ attenuator. Six calibration types were investigated: PIMMS TRL, Firmware TRL, SOLT, SOLR-Flush Thru, SOLR-Reciprocal Thru, and ECAL. The repeatability analysis was based on calculations of the experimental standard deviation of six repeated measurements at each frequency for each DUT and for each calibration type to establish the variability in the measurement data. The analysis was performed separately for both the magnitude and phase components of the measured complex-valued reflection and transmission coefficients.

The S-parameter results showed relatively good agreement between the different calibration techniques. The averaged differences from PIMMS for $S_{21}$ magnitude and phase are less than $2 \mathrm{mU}$ and $0.37^{\circ}$, respectively, for all calibration methods. The experiment standard deviation of S-parameter measurements is also similar for all calibration types. The experimental standard deviation of S-parameter magnitude and phase averaged over the frequency range is less than $1.5 \mathrm{mU}$ and $0.1^{\circ}$ for the measured reflection coefficients. From all the results it was seen that the experimental standard deviation for all calibrations is considerably smaller than the relative differences with respect to the reference PIMMS measurements. This shows that the random errors, the subject of this investigation, are significantly smaller than the other error types associated with S-parameter measurements.

The results from this investigation show the typical random errors from different calibration methods and for different types of DUT. These results can be used as a guideline for the random errors likely to be found in microwave S-parameter measurements made using other similar VNAs since the investigation has covered a wide range of devices and calibration types.

\section{ACKNOWLEDGMENTS}

The authors would like to thank Mr. James Skinner (NPL) for measuring the DUTs using PIMMS. The work described in this report was funded through the European Metrology Programme for Innovation and Research (EMPIR) Project JRP g04 'Metrology for advanced energy-saving technology in nextgeneration electronic applications.' The EMPIR is jointly funded by the EMPIR participating countries within EURAMET and the European Union.

\section{REFERENCES}

[1] N M Ridler, "A review of existing national measurement standards for RF and microwave parameters in the UK", IEE Colloquium Digest, No 99/008, pp 6/1 - 6/6, February 1999

[2] "IEEE Standard for Precision Coaxial Connectors (DC to $110 \mathrm{GHz}$ )", IEEE Std 287-2007

[3] G F Engen and C A Hoer, "Thru-Reflect-Line: An Improved Technique for Calibrating the Dual Six-Port Automatic Network Analyzer", IEEE Transactions on Microwave Theory and Techniques, Vol. 27, No. 12, pp 987 - 993, December 1979

[4] S Rehnmark, "On the calibration process of automatic network analyzer systems", IEEE Transactions on Microwave Theory and Techniques, Vol. 22, No. 4, pp 457-458, April 1974

[5] A Ferrero and U Pisani, "Two-port network analyser calibration using an unknown thru", IEEE Microwave and guided wave letters, Vol. 2, No. 12, pp 505-507, December 1992

[6] V Adamain "A novel procedure for network analyzer calibration and verification", $41^{\text {st }}$ ARFTG Conference, Spring 1993

[7] JCGM 100:2008, "Evaluation of measurement data - Guide to the expression of uncertainty in 
measurement", First Edition, September 2008. Available at: www.bipm.org.

[8] R W Beatty, "Calculated and Measured S11, S21, and group delay for simple types of coaxial and rectangular waveguide 2-port standards”, NBS Technical Note 657, December 1974 